\title{
Measuring ion velocity distribution functions in a compact, expanding helicon plasma
}

\author{
Daniel J. Lewis \\ West Virginia University
}

Follow this and additional works at: https://researchrepository.wvu.edu/etd

\section{Recommended Citation}

Lewis, Daniel J., "Measuring ion velocity distribution functions in a compact, expanding helicon plasma" (2008). Graduate Theses, Dissertations, and Problem Reports. 2610.

https://researchrepository.wvu.edu/etd/2610

This Thesis is protected by copyright and/or related rights. It has been brought to you by the The Research Repository @ WVU with permission from the rights-holder(s). You are free to use this Thesis in any way that is permitted by the copyright and related rights legislation that applies to your use. For other uses you must obtain permission from the rights-holder(s) directly, unless additional rights are indicated by a Creative Commons license in the record and/ or on the work itself. This Thesis has been accepted for inclusion in WVU Graduate Theses, Dissertations, and Problem Reports collection by an authorized administrator of The Research Repository @ WVU. For more information, please contact researchrepository@mail.wvu.edu. 


\title{
Measuring Ion Velocity Distribution Functions in a Compact, Expanding Helicon Plasma
}

\section{Daniel J. Lewis}

\author{
Thesis submitted to the \\ Eberly College of Arts and Sciences \\ at West Virginia University \\ in partial fulfillment of the requirements \\ for the degree of
}
Master of Science
in Physics

\author{
Earl E. Scime, Ph.D., Chair \\ Boyd Edwards, Ph.D. \\ Martin Ferer, Ph.D. \\ Department of Physics \\ Morgantown, West Virginia \\ 2008
}

Keywords: Laser Induced Fluorescence, Ion Velocity Distribution Function, Plasma Physics, Helicon source 


\section{Abstract \\ Measuring Ion Velocity Distribution Functions in a Compact, Expanding Helicon Plasma}

\section{Daniel J. Lewis}

Laser Induced Fluorescence (LIF) measurements of ion velocity distribution functions (ivdf) in a compact, expanding helicon plasma were limited by optical access and laser power. LIF measurements in the Compact HElicon Waves and Instabilities Experiment (CHEWIE) determined the ivdf of a compact helicon argon plasma as a function of fill pressure, magnetic field, and partial pressure of argon. The LIF scheme used the ring dye laser to $611.662 \mathrm{~nm}$ (vacuum wavelength) to pump the $\mathrm{Ar}$ II $3 \mathrm{~d}^{2} \mathrm{G}_{9 / 2}$ metastable state to the $4 p^{2} F_{7 / 2}$ state. The ion decayed to the $4 s^{2} D_{5 / 2}$ level and the fluorescent emission at $460.96 \mathrm{~nm}$ was recorded as a function of laser frequency. RF power up to $450 \mathrm{Watts}$ created steady state plasma in a $60 \mathrm{~cm}$ long, $7.5 \mathrm{~cm}$ diameter Pyrex tube source chamber. A $30 \mathrm{~cm}$ long, $15 \mathrm{~cm}$ diameter stainless steel expansion chamber was connected to one end of the source chamber. The magnetic field strength ranged from $0-550$ Gauss. Argon ivdfs indicate the presence of an accelerated ion population for fill pressure less than 1.5 mTorr. 


\section{Acknowledgments}

I would like to thank Dr. Earl Scime for his guidance throughout the project as thesis advisor and the support he gave me in the lab. I thank Robert Hardin for helpful conversations in the lab. I want to thank Dr. Mark Koepke for all the information provided in his plasma class. This has helped me in writing my thesis. I appreciate suggestions and discussions from Saeid Houshmandyar, Ioana Biloiu, Steve Przybysz, and Zane Harvey. I would like to thank Dr. Alex Hansen for his assistance in the lab and on the project. I appreciate the help Saikat Chakraborty Thakur provided me. I thank Dr. Costel Biloiu for helping me adjust to the lab. I would like to thank Carl Weber, Doug Mathess, and Tom Milam for their help in designing and building the front panel of CHEWIE. I appreciate the support and help that Sherry Puskar and Siobhan Byrne provided me in the administrative tasks. I thank Mike Zimmermann and Jeff Walker for useful physics discussions and for playing weekly squash games. I appreciate all that my parents have done for me and their love. 


\section{Table of Contents}

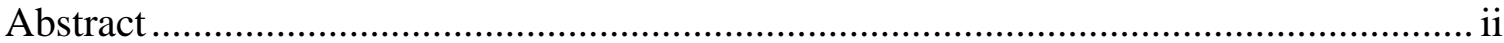

Acknowledgments............................................................................................... iii

List of Figures ...................................................................................................... v

List of Symbols .................................................................................................. vi

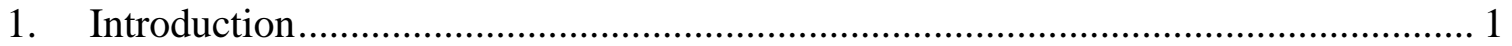

2. Principle of Measurements ............................................................................... 9

2.1. Langmuir Probe ......................................................................................... 9

2.2. Laser Induced Fluorescence.............................................................. 14

3. Experimental Setup..................................................................................... 22

4. Experimental Results ...................................................................................... 27

4.1 Langmuir Probe Measurements ...................................................................... 27

4.2 Laser Induced Fluorescence Measurements ....................................................... 34

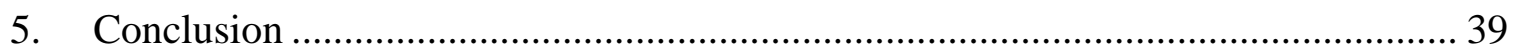

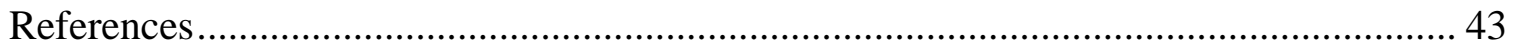




\section{List of Figures}

Figure 1 Diagram of a Double Layer ........................................................................ 4

Figure 2 Diagram of electric potential versus position.............................................. 5

Figure 3 Phase diagram of ion velocity versus position. ........................................... 6

Figure 4 Phase diagram of electron velocity versus position... .................................... 6

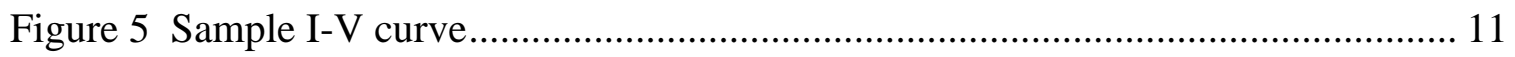

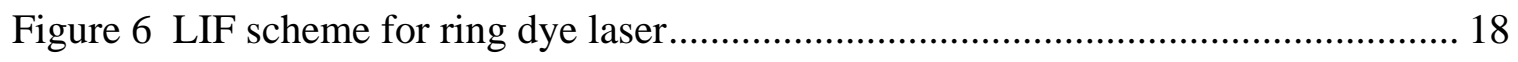

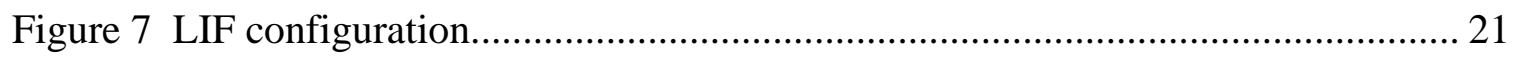

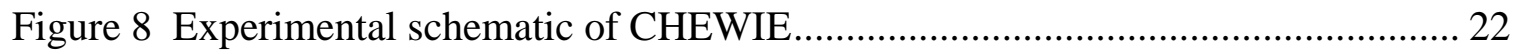

Figure 9 CHEWIE magnetic field profile .......................................................... 24

Figure 10 Electron density and electron temperature as a function of position............... 28

Figure 11 Electron density and electron temperature as a function of RF power........... 29

Figure 12 Electron density and electron temperature as a function of fill pressure ........ 31

Figure 13 Electron density as a function of electromagnet current ............................. 32

Figure 14 Electron temperature as a function of electromagnet current....................... 33

Figure 15 Langmuir probe I-V curve for maximum current in magnet 2 and magnet 3.34

Figure 16 LIF intensity versus laser frequency at a pressure of 2.5 mTorr .................... 36

Figure 17 LIF intensity versus laser frequency at a pressure of 1.2 mTorr ................... 37

Figure 18 LIF intensity versus laser frequency at a pressure of 1.2 mTorr .................... 38 


\section{List of Symbols}

$e$ - electron charge $\left(1.6 * 10^{-19} \mathrm{C}\right)$

$q_{i}$ - ion charge

$A_{p}$ - collection area of the probe

$m_{i}-$ ion mass

$M-$ argon ion mass

$m_{p}-$ proton mass

$m_{e}-$ electron mass

$T$ - temperature

$T_{e}$ - electron temperature

$T_{f e}$ - free electron temperature

$T_{t e}$ - trapped electron temperature

$T_{i}$ - ion temperature

$T_{f i}-$ free ion temperature

$T_{t i}$ - trapped ion temperature

$\kappa-$ Boltzmann constant $=\left(1.6 * 10^{-19} \mathrm{~J} / \mathrm{V}\right)$

$V_{p}$ - space potential

$V_{f}$ - float potential

$V$ - probe potential

$\varphi_{0}-$ potential drop

$\Delta v=2 \pi \Delta \omega-$ change in frequency

$v$ - laser frequency

$v_{o}=2 \pi \omega_{0}-$ rest frame frequency 


$$
\begin{aligned}
& \lambda-\text { wavelength } \\
& \Delta \lambda \text { - change in wavelength } \\
& I\left(v_{o}\right)-\text { intensity at rest frequency } \\
& I(v) \text { - intensity at laser frequency } \\
& v_{i t h}-\text { ion thermal velocity } \\
& v_{e}-\text { electron thermal velocity } \\
& v_{m i n}-\text { minimum velocity } \\
& \left.c-\text { speed of light ( } 3 * 10^{8} \mathrm{~m} / \mathrm{s}\right) \\
& u_{s}-\text { ion acoustic velocity }\left(\kappa T_{e} / m_{i}\right)^{1 / 2} \\
& n_{0}-\text { plasma density } \\
& \left.u_{B}-\text { Bohm velocity ( } \kappa T_{e} / m_{i}\right)^{1 / 2} \\
& n_{\omega}-\text { ion velocity } \\
& \omega_{c i}-\text { ion cyigma } \\
& \omega_{c e}-\text { electron cyclotron frequency } \\
& \lambda_{c}-\text { critical current density } \\
& n_{p e}-\text { electron plasma frequency }
\end{aligned}
$$




$$
\begin{aligned}
& u_{D i}-\text { ion drift velocity } \\
& u_{D e} \text { - electron drift velocity } \\
& \gamma \text { - adiabatic constant } \\
& \pi-\mathrm{pi} \\
& I_{s i}-\text { ion saturation current } \\
& L \text { - angular momentum } \\
& m \text { - magnetic quantum number } \\
& \Delta E \text { - uncertainty in energy } \\
& \Delta E_{n l m} \text { - Energy shift for Zeeman splitting } \\
& \Delta t \text { - uncertainty in time } \\
& \text { Ђ - Planck’s constant } \\
& \omega_{D} \text { - Doppler broadening parameter } \\
& L_{D}(\omega) \text { - Shape of line } \\
& \lambda_{V A C} \text { - vacuum wavelength } \\
& \lambda_{\text {AIR }} \text { - air wavelength } \\
& \varepsilon_{0} \text { - permittivity of free space }-8.8510^{-12} \mathrm{C}^{2} / \mathrm{Nm}^{2} \\
& \mu_{0} \text { - permeability of free space }-4 \pi 10^{-7} \mathrm{~N} / \mathrm{A}^{2} \\
& g \text {-dimensionless magnetic moment } \approx 2 \\
& \text { Ma - Mach number } \\
& \sigma=10^{4} / \lambda
\end{aligned}
$$




\section{Introduction}

Plasma, the fourth state of matter, is a partially ionized gas with free electrons and free ions. Gaseous plasma can be generated by applying an electric field to a gas. The electric field will cause an ionizing collision between electrons and neutral particles which results in free electrons and ions. There are four criteria which the plasma must satisfy: 1) quasi-neutrality, 2) Debye length, 3) bulk interaction, and 4) plasma frequency [Dendy, 1990; Hastings and Garrett, 2004]. Quasi-neutrality states that the electron density and ion density are approximately equal over a distance of Debye lengths. A Debye length is the shielding distance for a charged particle which means that the particles are close so that they may influence each other. The equation defining Debye length is

$$
\lambda_{D}^{2}=\varepsilon_{0} \kappa T_{e} /\left(n_{e 0} e^{2}\right)+\varepsilon_{0} \kappa T_{i} /\left(n_{i 0} q_{i}^{2}\right)
$$

where $\lambda_{D}$ is the Debye length, $\varepsilon_{0}$ is permittivity, $T_{e}$ is the electron temperature, $T_{i}$ is the ion temperature, $\kappa$ is the Boltzmann constant, $n_{e 0}$ is the electron density, $n_{i 0}$ is the ion density, $e$ is the electronic charge, and $q_{i}$ is the ion charge. The size of the Debye length is of the order of micrometers. For distances less than a Debye length, quasi-neutrality may be violated. For bulk interaction, the size of the quasi-neutral plasma is large compared to the Debye length. The electron plasma frequency, $\omega_{p e}^{2}=n_{e 0} e^{2} /\left(\varepsilon_{0} m_{e}\right)$, is large compared to electron neutral collision frequency.

The Compact HElicon Waves and Instabilities Experiment (CHEWIE) is designed to study the plasma at a low temperature and low pressure. The plasma is non-thermal since the temperature of the electron, ions, and neutrals are not equal. The electron temperature is large compared to the ion and neutral temperatures. There are 
three operational modes for the plasma source: 1) capacitively coupled plasma (CCP), 2) inductively coupled (ICP), and 3) helicon mode [Clarycon Co.]. The density of the plasma changes depending upon the mode of the plasma.

The capacitively coupled plasma has low to medium density plasma and requires large radiofrequency (RF) amplitude voltages between the plasma [Clarycon Co.]. In the capacitively coupled regime, there is high energy ion bombardment. Also, the space potential is modulated due to periodic electron current flow to the electrode. The plasma density for the capacitive mode ranges from $10^{9} \mathrm{~cm}^{-3}$ to $10^{10} \mathrm{~cm}^{-3}$ and the pressure ranges from 10 to 100 mTorr. In capacitively coupled plasmas, ohmic heating is dominant when the electron-neutral collision frequency is small. Stochastic heating is dominant due to electron reflection from the sheath surface in high frequency capacitively coupled plasmas. The collisions between electrodes are random in stochastic processes. CCP tends to have a uniform plasma density. The color of the capacitively coupled argon plasma is pink and dim. The plasma is weakly ionized in this mode and the electrostatic field dominates.

The inductively coupled mode has higher density plasmas and requires large RF current between the coils and the plasma. Ion bombardment energy is much smaller in the inductive mode compared to the capacitive mode. The plasma is sustained by time varying current which causes a time varying magnetic field. Then, the time varying magnetic field induces a time varying electric field. In this mode, the induced electric field tends to dominate the electrostatic field. ICP is used in the generation of free radicals in ion generators, and in atmospheric plasma torches [Vinogradov, 2003]. ICP is also used in plasma processing. The advantage of ICP over CCP is that it requires low 
RF power, low gas pressure, and yields a higher density. The color of the argon plasma in the inductively coupled mode is purple and the electron density is $10^{11} \mathrm{~cm}^{-3}$ [Yin et al, 1999].

The helicon mode of the plasma has even higher density than the inductive mode. In this mode, a whistler wave appears since it is a solution which satisfies the dispersion relation $k_{\omega}$

$$
k_{\omega}^{2}=\omega \omega_{p}^{2} / \omega_{c e} c^{2}=\omega n_{0} e \mu_{0} / B_{0}
$$

where $\omega_{c e}$ is the electron cyclotron frequency and $\omega_{p}$ is the plasma frequency [Chen and Boswell, 1997]. A right circularly polarized electromagnetic wave propagates in the helicon mode at a frequency of $\omega_{c i}<<\omega<<\omega_{c e}$, where the ion cyclotron frequency is $\omega_{c i}=q_{i} B / m_{i}$ and the electron cyclotron frequency is $\omega_{c e}=q_{e} B / m_{e}$. Helicon experiments are traditionally performed with cylindrical geometry and an antenna surrounding the cylindrical chamber. An RF electromagnetic wave is applied to a gas to create the plasma, which is "electrodeless" [Maurice et al, 2002]. In this mode, the color of the argon plasma is an intense blue. Helicon discharges can achieve nearly $100 \%$ ionization, thus there can be few neutral gas particles present [Chen and Boswell, 1997].

A sheath is formed as a result of an object inserted into the plasma or a physical boundary such as a wall, except when the object is at space potential. The sheath's size is of the order of a few Debye lengths. There are three parts to the plasma: the sheath, the pre-sheath (transitional region), and the quasi-neutral plasma [Merlino, 2007]. In the transitional region, there is a small change in the electric field over a large distance. This distance tends to be large compared to the Debye length and the sheath. The pre-sheath is the region where ions are accelerated to at least the Bohm velocity $-u_{B}=\left(k T_{e} / m_{i}\right)^{1 / 2}$. 
In the sheath, quasi-neutrality is violated due to an excess of ions in this region [Sternberg and Godyak, 2007]. There is also a large potential drop in the sheath region. The sheath forms in order to maintain quasi-neutrality throughout the bulk plasma [Merlino, 2007].

A double layer (DL) is a region of two equal but oppositely charged layers that are parallel, as shown in Figure 1 [Block, 1978]. As a result, the inertia terms balance the electrostatic forces. There are three properties a double layer needs to have present. The first property, the potential drop, $\phi_{0}$ is $\left|\phi_{0}\right|>\kappa T / e$ where $T$ is the temperature of the coldest plasma species bordering the double layer, $\kappa$ is Boltzmann constant, and $e$ is the electronic charge. The second property is the strong electric field in the double layer which causes the positive and negative charges to cancel each other out. The third property states that quasi-neutrality is locally violated in both layers. The typical electric-field aligned dimension of a DL is from ten Debye lengths to one hundred Debye lengths.

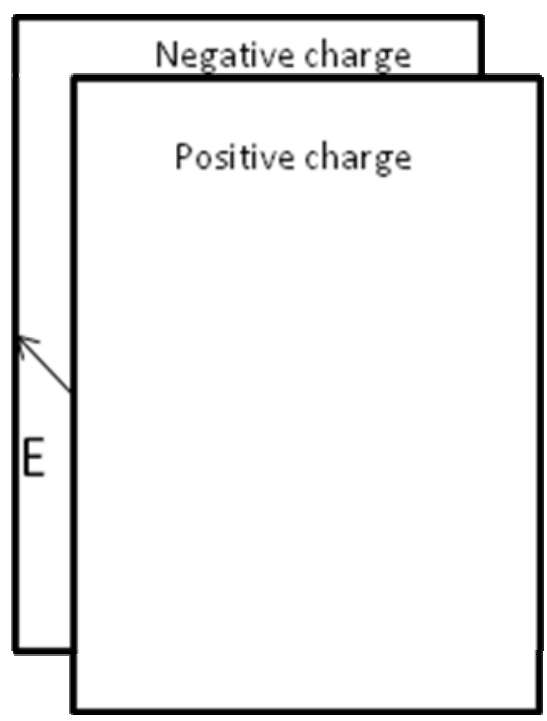

Figure 1 Diagram of a Double Layer 
A double layer has four charged species populations in the plasma which explains the potential drop. The particles present are: free electrons, free ions, trapped or thermal electrons, and trapped or thermal ions. The potential of the double layer and the behavior of the electrons and ions are shown in Figures 2, 3, and 4. The trapped particles are treated as the background stationary plasma, while the free particles are considered beams. A beam is a group of charged particles moving one direction and faster than the background population. The trapped electrons remain on the high potential side of the double layer, while the trapped ions stay on the low potential side of the double layer. The trapped particles are reflected in its own motion. The free electrons accelerate from the low potential side to the high potential side of the double layer, while the free ions accelerate from the high potential side to the low potential side of the double layer.

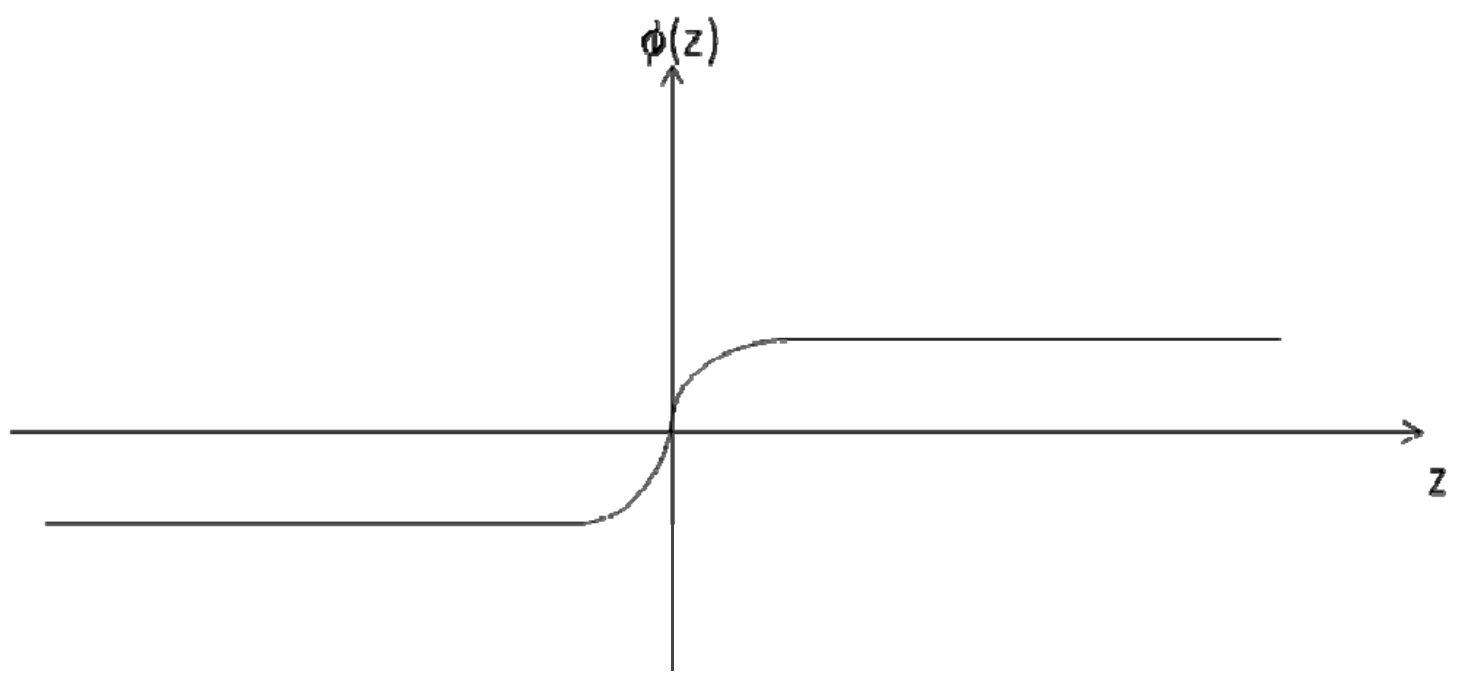

Figure 2 Diagram of electric potential versus position 


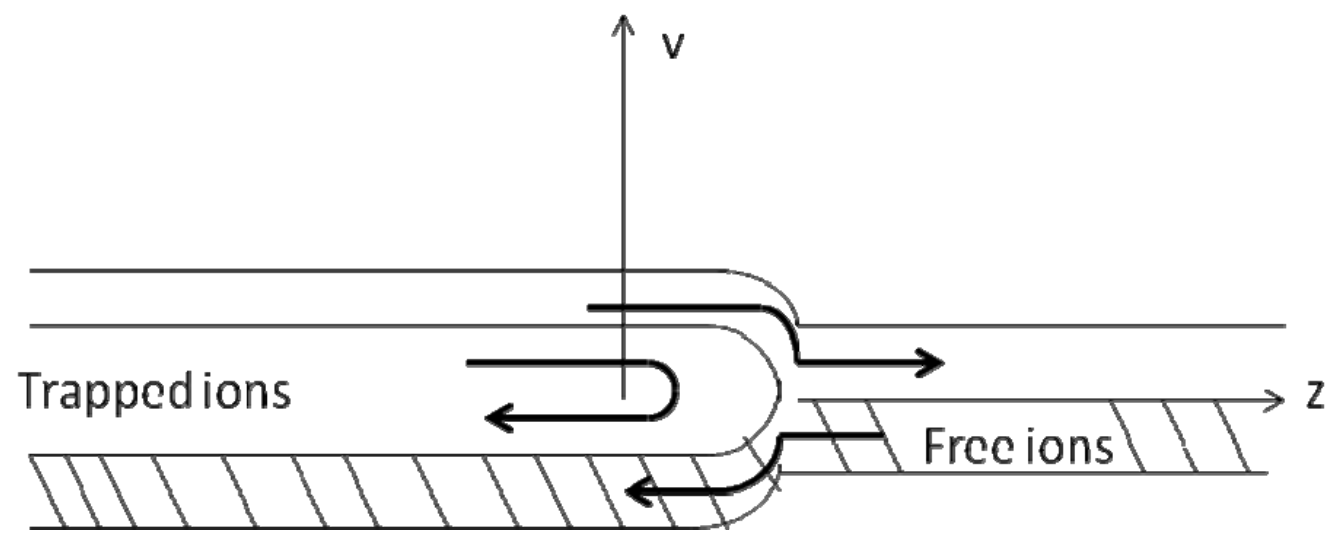

Figure 3 Phase diagram of ion velocity versus position. Note that the trapped ions move in the positive $z$ direction. The trapped ions are reflected and move in the negative $z$ direction. The free ions (beam) start at positive $z$ and move to negative $z$.

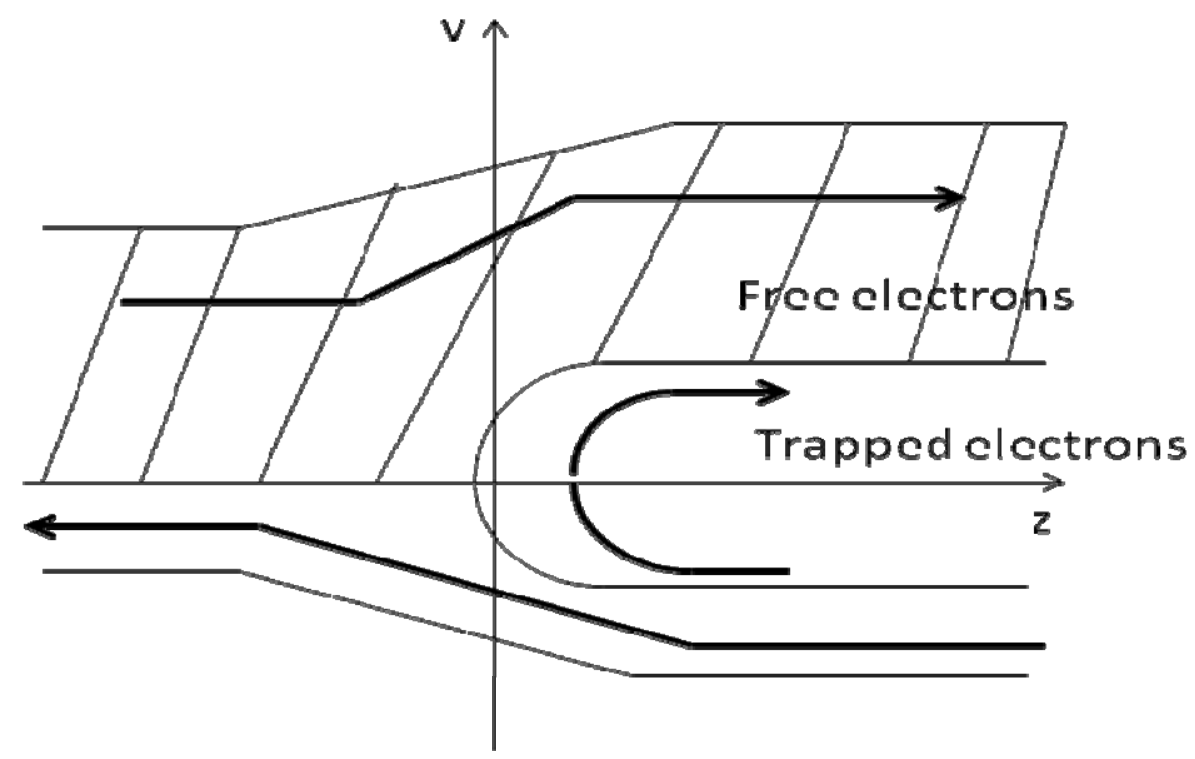

Figure 4 Phase diagram of electron velocity versus position. The trapped electrons begin moving in the negative $z$ direction. Then, they are reflected and move in the positive $z$ direction. The free electrons (beam) start at negative $z$ and move in the positive $z$ direction.

The Langmuir criteria explain the strong electric field in the double layers. The Bohm criteria describe how quasi-neutrality is violated in the double layers. In order to satisfy the Langmuir criteria, the flux of electrons through the layer must be much larger than the flux of the ions since the electrons have a much smaller transit time in the DL. 
For strong double layers, it is possible to have a net current. Each charged species is accelerated through the double layer potential so that

$$
\left(n_{e} u_{D e}\right) /\left(n_{i} u_{D i}\right)=\left(m_{i} / m_{e}\right)^{1 / 2}
$$

where $u_{D e}$ is the electron drift velocity and $u_{D i}$ is the ion drift velocity [Block, 1978]. Due to the Langmuir criteria, the ion density must decrease faster than the electron density. Specifically, $n_{i} \sim \exp \left(-e \phi / k T_{t i}\right)$ and $n_{e} \sim u^{-1}{ }_{D e}=\left(u_{D e 0}^{2}+2 e \phi / m_{e}\right)^{-1 / 2}$ with $u_{D e 0}=u_{D e}$ at $\phi=0$ describes the Bohm criteria. The double layer has both negative and positive boundaries. At the negative boundary, free electrons are entering the double layer with a drift velocity of $u_{D e}$ and have the same density as the trapped ions. The trapped electrons and free ions have small densities at the negative boundary. As the potential is increased from the negative boundary to the positive boundary, the electron and ion densities decrease. At the positive boundary, the free ions are entering the double layer with a drift velocity of $u_{D i}$ and have the same density as trapped electrons. The trapped ions and free electrons have negligible densities at the positive boundary.

For a double layer to form, the Bohm criteria states that the minimum current density is

$$
j_{c}=n_{0} e\left(2 k T_{e} / m_{e}\right)^{1 / 2}
$$

where $j_{c}$ is the critical current density. The Bohm criteria describes the minimum electron drift velocity when entering the double layer which is

$$
m_{e} u^{2}{ }_{D e 0}=\kappa\left(\gamma T_{f e}+T_{t i}\right)
$$

where $\gamma$ is the adiabatic constant for free electrons, $T_{f e}$ is the free electron temperature, and $T_{t i}$ is the trapped ion temperature. Simulations and experiments by Goertz and Joyce 
[1975] show that the kinetic energy of the electron is larger than the thermal energy of free electrons, $m_{e} u_{D e 0}^{2}>2 \kappa T_{f e}$.

There are three regimes where double layers appear, which are associated with the velocity of the particles [Quon and Wong, 1976]. In regime one, the electron drift is $0.3 v_{e}>u_{D e}>c_{s}$ where $v_{e}$ is the electron thermal velocity, $u_{D e}$ is the electron drift, and $c_{s}$ is the ion acoustic speed. The electron thermal velocity is defined as $v_{e}=\left(k T_{e} / m_{e}\right)^{1 / 2}$. Current driven ion acoustic instabilities are observed to grow spatially which saturate due to an electron trapping process. In regime two, the electron drift is of the order of the electron thermal velocity. The two stream instability is observed. The potential double layer is stable with a change in potential of 3 to $15 \mathrm{~V}$ over a distance of 3-5 cm $\left(20-30 \lambda_{D}\right)$. The drift velocities would range from $v_{e} \leq u_{D e} \leq 3 c_{s}$. In regime three, the electron drift velocity is from $u_{D e}=3-10 v_{e}$. The electron beam velocity distribution drives electron plasma waves to large amplitudes and excites ion waves. With an intense high frequency field the beam achieves thermal equilibrium which causes the double layer to break up into intense field spikes. The result is an unstable double layer and plasma turbulence [Quon and Wong, 1976]. 


\section{Principle of Measurements}

\subsection{Langmuir Probe}

Langmuir probe was the first technique used to determine the electron density and electron temperature of the plasma. A Langmuir probe is a conductor inserted into the plasma which perturbs the plasma over a small region. The conductor is biased over a range of voltages and the current is determined at each voltage. The space potential is the electric potential of the plasma, while the floating potential is the potential at which the probe collects zero current. A common misconception is when the Langmuir probe is placed inside of the plasma, the probe is at the space potential. Instead, the probe is at the floating potential [Merlino, 2007]. The floating potential is more negative than the space potential. A probe reaches the floating potential by initially collecting more electrons than ions since electrons have a smaller mass, but a greater velocity. The electron flux to the probe increases with a larger electron velocity [Merlino, 2007]. At space potential, there is no potential difference between the probe and the plasma which allow charged particles to travel toward the probe without a change in its potential. Also, the space potential corresponds to an inflection point or "knee" of an IV curve where the second derivative is zero, as shown in Figure 5. Not all knees correspond to the space potential. Certain knees can indicate the presence of a beam population [Ruzic, 1994]. The potential difference between the space potential and floating potential predominantly occurs over the sheaths and less in the pre-sheath.

An I-V plot shows the current collected as a function of biased voltage. There are four regions which are important in order to analyze an I-V curve. In the first region, the probe potential is much larger than the space potential which causes the probe to function 
like an anode. In the second region, the potential is above the space potential, but less than the potential in the first region. The current collected increases slightly from the space potential until saturation. The electron current dominates in the electron saturation region. The third region corresponds to the electron retardation region. The probe potential is greater than the floating potential, but less than the space potential. The electron current behaves as an exponential function for a Maxwell-Boltzmann velocity distribution of electrons [Merlino, 2007]. Electrons with less than the minimum velocity are repelled in this region, while the fast electrons still reach the probe. The minimum velocity of the electron required to reach the probe is $0.5 * m v^{2}{ }_{\min }=e\left(V_{p}-V\right)$. In the fourth region, the probe potential is less than the floating potential. The probe is negatively biased and only collects ions. When the ion enters the sheath, it is actually accelerated to a velocity of $u(x)=u_{0}\left(1-2 e V(x) / m_{i} u_{0}^{2}\right)^{1 / 2}$. In the ion saturation region, the ion current is approximately constant over a large change in voltage [Merlino, 2007]. 


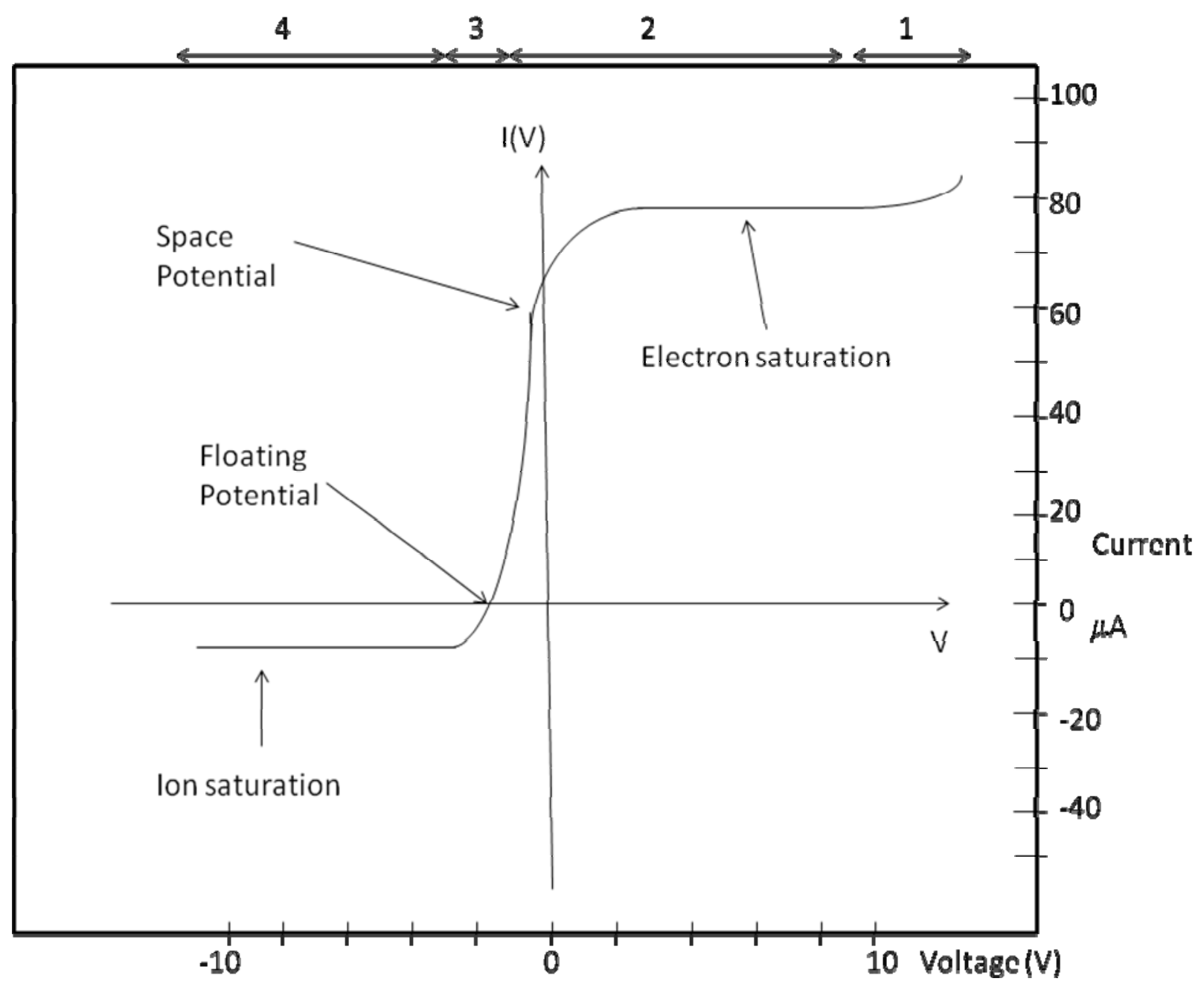

Figure 5 Sample I-V curve. In region 1, the Langmuir probe functions as an anode. Region 2 shows electron saturation. In region 3 , electron retardation corresponds to the probe potential between the space potential and floating potential. Region 4 illustrates ion saturation.

It is generally desirable to operate the Langmuir probe near the floating region of the I-V curve since the current is small. When operating in the electron saturation region, it is possible to destroy the probe or disrupt the plasma due to the high current. In the electron saturation region, the current is approximately the same as the random electron current across the probe's area. Random current is current unassociated with the probe bias. 
When the electron temperature is much larger than the ion temperature $\left(T_{e}>>T_{i}\right)$, the ion saturation current for an unmagnetized plasma is $I_{s i}=0.6$ e $n_{i} v_{i}$ th $A_{\text {probe }}$ where $v_{i t h}=\left(\kappa T_{e} / m_{i}\right)^{1 / 2}$ can determine the ion density. In magnetized plasma, the previous formula for the ion saturation can be calculated with 0.49 instead of 0.6 [Keesee, 2007]. The magnetized plasma ion saturation current uses the approximation that $0.49 \approx 0.5$. The reason the ion temperature is much less than the electron temperature is due to the fact that the neutrals are cold relative to the electrons. Both an ion and a free electron are created by an ionizing collision between an electron and a neutral. The collision does not transfer enough energy to the ion which makes the ion cold relative to the electron. The electric field accelerates the electron in a fixed direction which allows the electron to have a larger temperature. When the probe potential is between the floating potential and the space potential, the current collected by the probe is

$$
\begin{gathered}
I=(-1 / 2) e A n_{i} v_{i t h}+(1 / 4) e A n_{e}\left[8 \kappa T_{e} /\left(\pi m_{e}\right)\right]^{1 / 2} \exp \left(-e\left(V_{p^{-}} V\right) / \kappa T_{e}\right) \\
\text { or } \\
I=I_{s i}+(1 / 4) e A n_{e}\left[8 \kappa T_{e} /\left(\pi m_{e}\right)\right]^{1 / 2} \exp \left(-e\left(V_{p^{-}} V\right) / \kappa T_{e}\right)
\end{gathered}
$$

where $V_{p}$ is the space potential. Since the space potential is not directly determined it can be calculated by solving Eq (6) iteratively. From the space potential, the electron temperature is determined. Once the electron temperature and ion saturation current are known, it is possible to determine the plasma density. The first term represents the ion saturation current. Next, Eq (6) is differentiated with respect to $\left(\mathrm{V}-\mathrm{V}_{\mathrm{p}}\right)$. The new equation becomes

$$
d I / d\left(V-V_{p}\right)=d I_{s i} / d\left(V-V_{p}\right)+\left(I-I_{s i}\right)\left(e / \kappa T_{e}\right)
$$


Assuming that $d I_{s i} / d\left(V-V_{p}\right)<<d I / d\left(V-V_{p}\right)$ and solving for electron temperature, Eq (8) becomes

$$
T_{e}=e\left(I-I_{s i}\right) /\left[\kappa d I / d\left(V-V_{p}\right)\right]
$$

In this experiment, graphite was used as a tip due to its high melting point. The cylindrical probe had a $2 \mathrm{~mm}$ long tip with a $0.5 \mathrm{~mm}$ diameter. The Langmuir probe was biased from -50 to $+40 \mathrm{~V}$ by a Keithley 2400 SourceMeter. This range of voltages included the electron retardation region and ion saturation region, but not the electron saturation region. 


\subsection{Laser Induced Fluorescence}

Plasma spectroscopy does not require detailed knowledge of the plasma due to the electromagnetic radiation or absorption. A line shape may have six different effects that change its width or spectral position: 1) Stark (pressure) broadening, 2) Natural line broadening, 3) Zeeman broadening, 4) Power (saturation) broadening, 5) Instrumental broadening, and 6) Doppler broadening [Griem, 1997]. Spectroscopic measurements deal with determining the quantity of atoms or molecules which absorb or emit electromagnetic radiation. Laser Induced Fluorescence (LIF) is one example of plasma spectroscopy. In LIF, the laser scans an absorption line of an ion or an atom in the plasma. Then, the fluorescent emission is measured as a function of laser frequency. The shape of the absorption line is determined and can be used to determine the ion velocity distribution function (ivdf) [Hill et al, 1983]. In LIF, the spectral line width is Doppler broadened by the velocity of its particles. If a particle is moving away from the laser light, then the laser will see the particle's frequency lower than its rest frequency. Therefore, the laser light needs to be at a higher frequency than its rest frequency. If the particle is moving toward the laser light, then the laser sees the particle's frequency higher than its rest frequency. The laser light needs to be at a lower frequency than its rest frequency.

The first effect is Stark (pressure) broadening, which can be problematic LIF in plasmas. In Stark broadening, either a wave or a group of particles generate an electric field which causes pressure broadening in plasma. Stark broadening tends to affect lines which allow electric dipole transitions [Griem, 1974]. In pressure broadening, it is usually the Stark term that dominates the Van der Waal's and resonance broadening. Ion 
produced fields cause lines to broaden and result in overlapping Stark broadening. Van der Waal's broadening occurs between a neutral perturber and radiator, which are of different species. Resonance, or self broadening, occurs between a radiator and perturber of identical species. In resonance broadening, the line width is independent of velocity [Griem, 1997]. When Stark broadening dominates Doppler broadening, the electron density can be determined, but not the temperature. Electron collisions result in the broadening of isolated lines [Griem, 1997].

The second effect is natural line broadening which is due to the Heisenberg uncertainty principle. Natural line broadening depends upon the energy-time uncertainty in quantum states where $\Delta E \Delta t \geq \hbar / 2$. States with longer lifetimes have less broadened compared to the states with a shorter lifetime. This occurs due to the fact that interactions with electromagnetic fields from photons cause an approximate eigen-mode system. The result of the energy distribution determines how the line shape is broadened. Spectral lines of singly ionized atoms appear in the visible, while multiple ionized atom spectral lines are in the vacuum ultraviolet. Natural line broadening is negligible with the exception of inner shell x-ray transitions, autoionizing levels, and resonance lines for highly charged ions [Griem, 1997]. Natural broadening is negligible compared to other processes such as Doppler broadening.

The third effect on the spectral line is Zeeman broadening in magnetized plasmas. The magnetic field splits $\mathrm{L}_{\mathrm{z}}$ into $(2 \mathrm{~L}+1)$ parts. The allowed transitions for angular momentum is $\Delta \mathrm{L}=1$ and $\Delta \mathrm{m}= \pm 1,0$ where $\Delta \mathrm{m}=0$ is the $\pi$ transition and $\Delta \mathrm{m}= \pm 1$ is the $\sigma$ transition. The $\pi$ transition has linearly polarized light and $\sigma$ transition has circularly polarized light [Griem, 1997]. The spectral lines split with a stronger magnetic 
field. The maximum $\pi$ component has a larger intensity than the $\sigma$ components. The energy shift for Zeeman splitting is $\Delta E_{n l m}=\left(e B^{*} \zeta * g * m\right) /\left(2 m_{e}\right)$ [Gasiorowicz, 2003]. Dividing by $h$ to calculate $\Delta v$, the shift in the centerline for the frequency change is

$$
\Delta v=\left(e^{*} B^{*} g^{*} m\right) /\left(4 \pi m_{e}\right)
$$

where $\Delta v$ is the shift of the line, and $m$ is the magnetic quantum number [Griem, 1974].

The fourth effect on the spectral line is power broadening due to high laser intensity in the plasma, which results in line saturation. When the stimulated photon emission equals the photon absorption rate and is greater than the spontaneous photon emission rate, the frequencies near the wings of the spectral line are broadened [Goeckner and Goree, 1989]. These three processes, spontaneous emission, stimulated emission, and absorption, can determine the strength of power broadening. Also, three different states $-0,1$, and 2 - are necessary to describe the processes. Absorption occurs when an atom or ion in a lower state, 0 , receives an adequate amount of energy to reach a higher excited state, 1 . Spontaneous emission occurs when an atom in an excited state, 1 , emits energy without an external influence and decays to a lower state, 2. Stimulated emission occurs when an atom or ion in an excited state, 1 , loses energy due to an interaction from an incoming photon and returns to the original state, 0 . Saturation broadening occurs when the laser has achieved a sufficient intensity. Above certain laser intensities, the absorption process saturates [Goeckner and Goree, 1989].

The fifth effect on the spectral line, instrumental broadening, is due to the laser line width or the light dispersing into the system. Instrumental broadening can be a problem that causes a line to appear Gaussian. Dispersion does not apply since a 
photomultiplier tube and a passband filter is used to detect the light [Boivin, 2000]. The laser bandwidth in our system is less than $1 \mathrm{MHz}$.

The sixth effect, the Doppler Effect, determines the temperature of a particular species. The temperature of a neutral particle, an ion, or an electron can be determined through its velocity distribution function by using the Full Width Half Maximum (FWHM). Generally, electrons are assumed to have Maxwellian distributions except in laser produced plasmas. Electron temperature is usually determined by comparing the ratio of the line intensities of the same atom. However, for an ion or neutral atom, the temperature is determined by measuring a one dimensional velocity distribution function along the lasers line of sight. The main assumption is that the Doppler Effects dominate the process of line broadening and that the particle is non-relativistic. The normalized line shape is Gaussian. The shape of the line is described as

$$
L_{D}(\omega)=\exp \left[-\left(\Delta \omega / \omega_{D}\right)^{2}\right] /\left(\pi^{1 / 2} \omega_{D}\right)
$$

where $L_{D}(\omega)$ is the shape of the line, $\Delta \omega$ is the change in frequency, and $\omega_{D}$ is the Doppler broadening parameter. The Doppler broadening parameter is

$$
\omega_{D}=\left(2 \kappa T / M c^{2}\right)^{1 / 2} \omega_{0}
$$

where $\omega_{0}$ is the laser's natural frequency, $\mathrm{M}$ is the mass of an argon ion $-40 * m_{p}$, and $m_{p}$ is the mass of a proton [Griem, 1997]. At full width half maximum, the right hand side (RHS) of Eq (12) should be multiplied by 1.665. The ion temperature can be calculated using equation 12. The line is Gaussian to at least $20 \%$ of its own intensity [Griem, 1997]. The thermal velocity of a beam can be calculated by

$$
v_{i t h}=\left(c \Delta v /\left(v_{0}(\ln 2)^{1 / 2}\right)\right.
$$


where $\Delta v$ is the change in frequency. The laser frequency is scanned over $10 \mathrm{GHz}$. The scanned wavelength is determined by taking the first derivative of

$$
c / \lambda=v
$$

The result is

$$
c \Delta \lambda / \lambda^{2}=-\Delta v
$$

Substituting equation 13 into equation 14 and taking the absolute value, the result becomes

$$
|\Delta \lambda / \lambda /=| \Delta v / v \mid
$$

The laser scans over a wavelength range of .012 $\mathrm{nm}$ by solving Eq (16).

Ar Il singly ionized argon LIF scheme for ring dye Laser

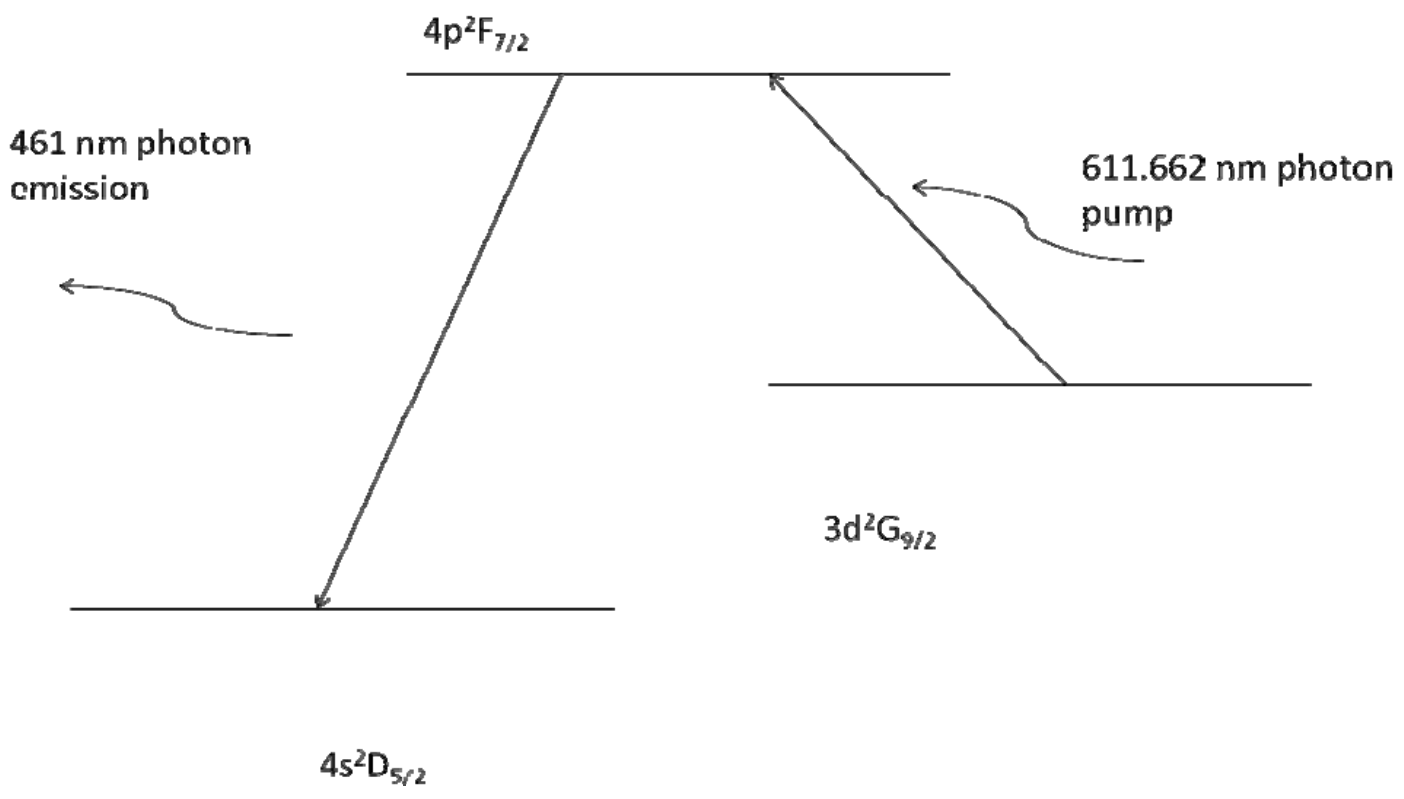

Figure 6 LIF scheme for ring dye laser

In the experiment performed on CHEWIE, a 3 level LIF scheme is used for the singly ionized argon ion, Ar II, as shown in Figure 6. The laser pumps the argon ion from $3 \mathrm{~d}^{2} \mathrm{G}_{9 / 2}$ metastable state to $4 \mathrm{p}^{2} \mathrm{~F}_{7 / 2}$ with a photon at vacuum wavelength 
$611.662 \mathrm{~nm}$. The corresponding air wavelength is $611.4923 \mathrm{~nm}$. Then, the ion decays to the $4 \mathrm{~s}^{2} \mathrm{D}_{5 / 2}$ and radiates a $460.96 \mathrm{~nm}$ photon. A metastable state has a larger ion population since it has a much longer lifetime. The formula for conversion from vacuum wavelength to air wavelength is given by

$$
\left(\lambda_{V A C}-\lambda_{A I R}\right) / \lambda_{A I R}=6.4328 \times 10^{-5}+\left(2.94981 \times 10^{-2} /\left(146-\sigma^{2}\right)\right)+2.5540 \times 10^{-4} /\left(41-\sigma^{2}\right)
$$

where $\lambda_{A I R}$ represents the air wavelength, $\lambda_{V A C}$ represents the vacuum wavelength, and $\sigma^{2}=10^{4} / \lambda$ in angstroms [Morton, 1991].

In this LIF system, a Coherent Innova 90 6-Watt argon-ion laser pumps a Coherent ring dye laser, model 899-21, to perform LIF measurements, as shown in Figure 7. The organic dye used is rhodamine G6 and its solvents are ethylene glycol and methanol. Rhodamine G6 is tunable over $560-640 \mathrm{~nm}$ and is a xanthene dye [Saleh and Teich, 1991]. The benefit of using a dye laser is that it is a tunable laser. Using liquid makes it possible to achieve a higher power output along with a larger range of available wavelengths. A ring dye laser is a Sagnac interferometer which uses a beam splitter splits the light into two components which travel in opposite directions to determine the velocity of particles. A beam splitter passes $10 \%$ of the light to the iodine cell and the remaining $90 \%$ to the CHEWIE apparatus. The iodine cell is viewed by an avalanche photodiode. An avalanche photodiode (APD) works by detecting a photon and cascading it into carrier pairs. Due to such a large gain, the APD can detect weak light signals [Saleh and Teich, 1991]. The iodine cell is used to measure the fluorescent spectrum of iodine to provide an absolute measurement of the laser wavelength. After passing through the iodine cell, the light is then coupled into a Burleigh WA 1500 wavemeter, 
which is used for coarse tuning of the wavelength. Before entering the CHEWIE apparatus, the remaining $90 \%$ of the light is modulated by an optical chopper at $1000 \mathrm{~Hz}$ and coupled into a fiber optic cable. Light is coupled into the injection optics where the laser light enters the plasma and travels along CHEWIE's z-axis which is parallel to the magnetic field. Next, the collection optics, a set of lens which focuses the light into a photomultiplier tube detector with a $1 \mathrm{~nm}$ passband filter centered at $461 \mathrm{~nm}$, receives the light signal from the plasma. A photomultiplier tube works on the photoelectric effect. Electrons are emitted from a cathode by a photon and then travel to the anode due to the potential difference. Secondary emissions lead to a cascade of electrons which cause amplification. As a result, the electron current is proportional to the photon flux [Saleh and Teich, 1991]. A Stanford model SR830 digital signal processor lock-in amplifier is used to differentiate the amplitude, phase, and frequency of the fluorescent signal from the noise. The reference input signal for the lock-in amplifier is the chopper. 


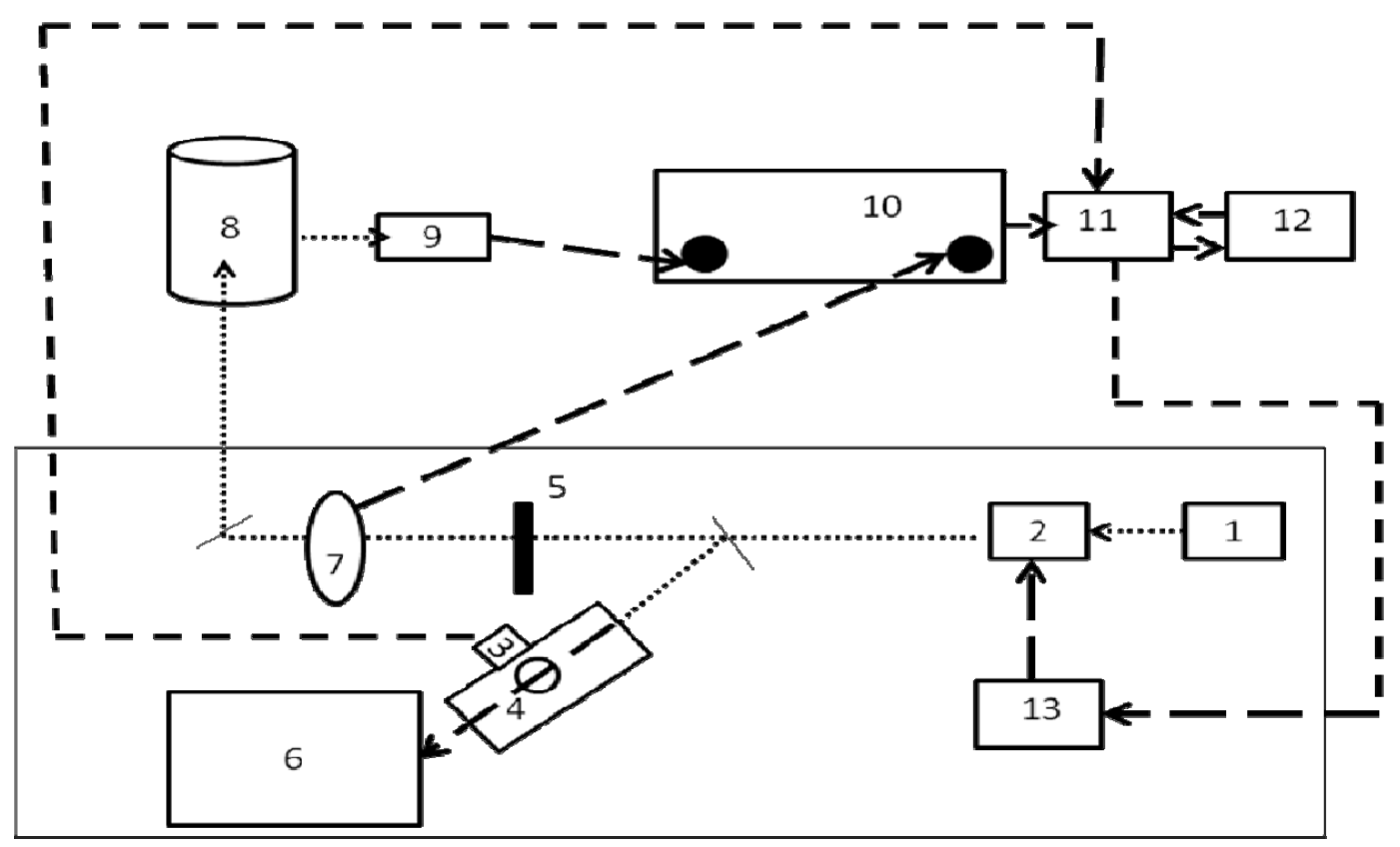

Figure 7 LIF configuration: 1) Argon ion laser; 2) Ring dye laser; 3) Avalanche photodiode; 4) $10 \%$ of the light passes through the iodine cell; 5) Power meter; 6) Wavemeter; 7) Optical chopper; 8) $90 \%$ of the laser light to plasma; 9) Bandpass filter and Photomultiplier tube; 10) Lock-in Amplifier; 11) I/O card; 12) Computer; 13) Laser Controller 


\section{Experimental Setup}

All the measurements were performed on the apparatus CHEWIE as shown in Figure 8. The source chamber is a cylindrical Pyrex glass tube 24 inches long and 3 inches in diameter. The expansion chamber is made of stainless steel and is $30 \mathrm{~cm}$ long and $15 \mathrm{~cm}$ in diameter. Gas flows from the top of the source chamber down to the expansion chamber. The top of the source chamber was air cooled by two fans. The Langmuir probe was inserted at the top port of the expansion chamber.

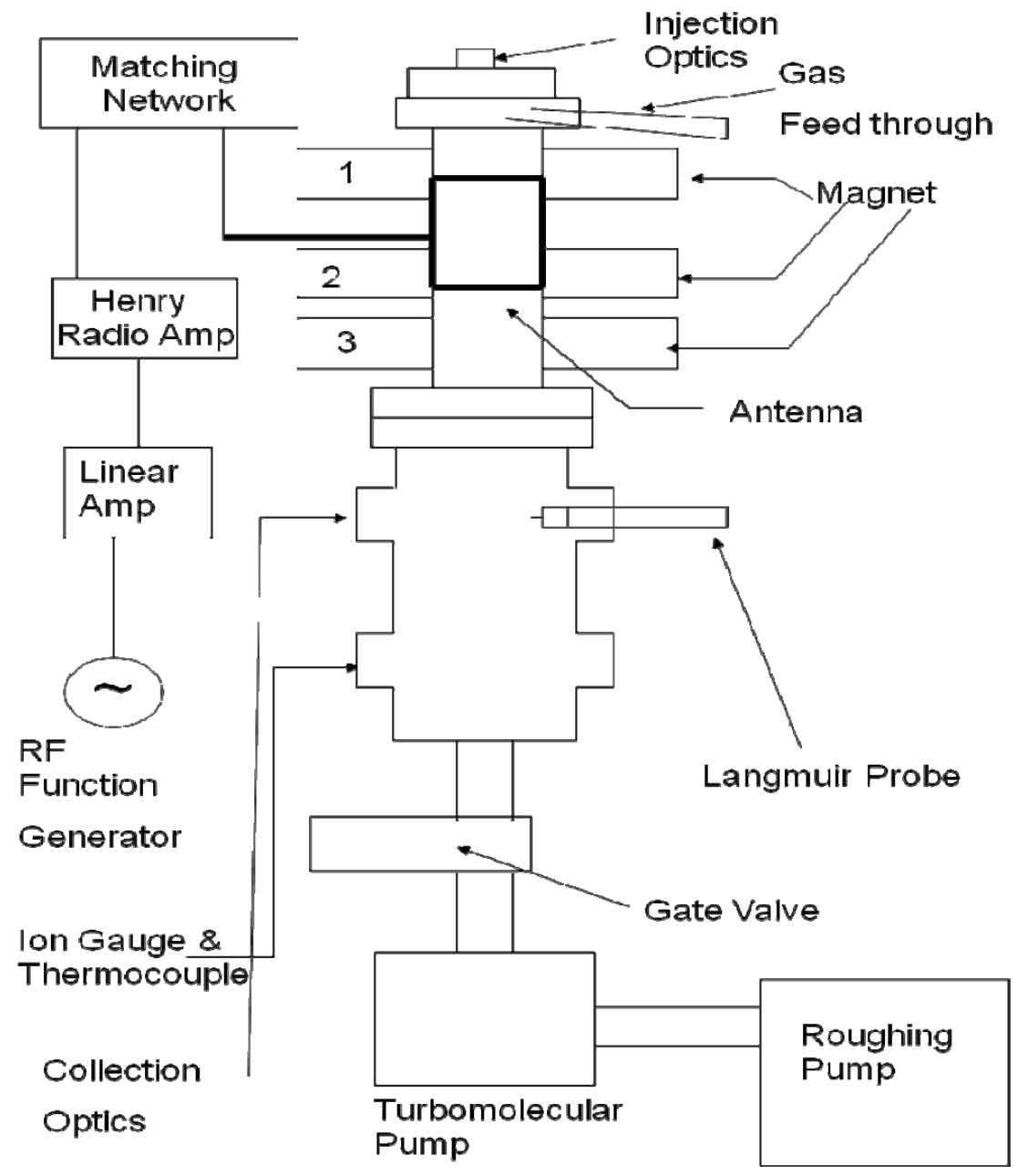

Figure 8 Experimental schematic of CHEWIE

The electromagnets were water cooled by an Accurate Gas Controller Systems chiller Model 708. Three electromagnets surrounded the source chamber. They are 
$10 \mathrm{~cm}$ long, with an inner radius of $5 \mathrm{~cm}$ and an outer radius of $17 \mathrm{~cm}$. The top of the source chamber corresponded to zero on CHEWIE's z axis. Magnet 1 was placed from $10.5 \mathrm{~cm}$ to $20.5 \mathrm{~cm}$ below the top of the chamber. Magnet 2 was placed from $32.8 \mathrm{~cm}$ to $42.8 \mathrm{~cm}$, while magnet 3 was placed from $47.3 \mathrm{~cm}$ to $57.3 \mathrm{~cm}$.

The Sorensen DCR 20-25B power supply provided 0 - 4 amps of current to magnet 1. A second power supply, a Sorensen DCR60-13 A, was used to power magnet 2 and magnet 3 in series. The second power supply provided 0 to 7 amps of current. A magnetic field profile of the experiment was measured using a Gauss meter every two centimeters. The first case had magnet 1 at 0 amps and magnet 2 and magnet 3 at 7 amps. The second case had magnet 1 at 4 amps and magnet 2 and magnet 3 at 7 amps. The magnetic field profile is shown in Figure 9. 


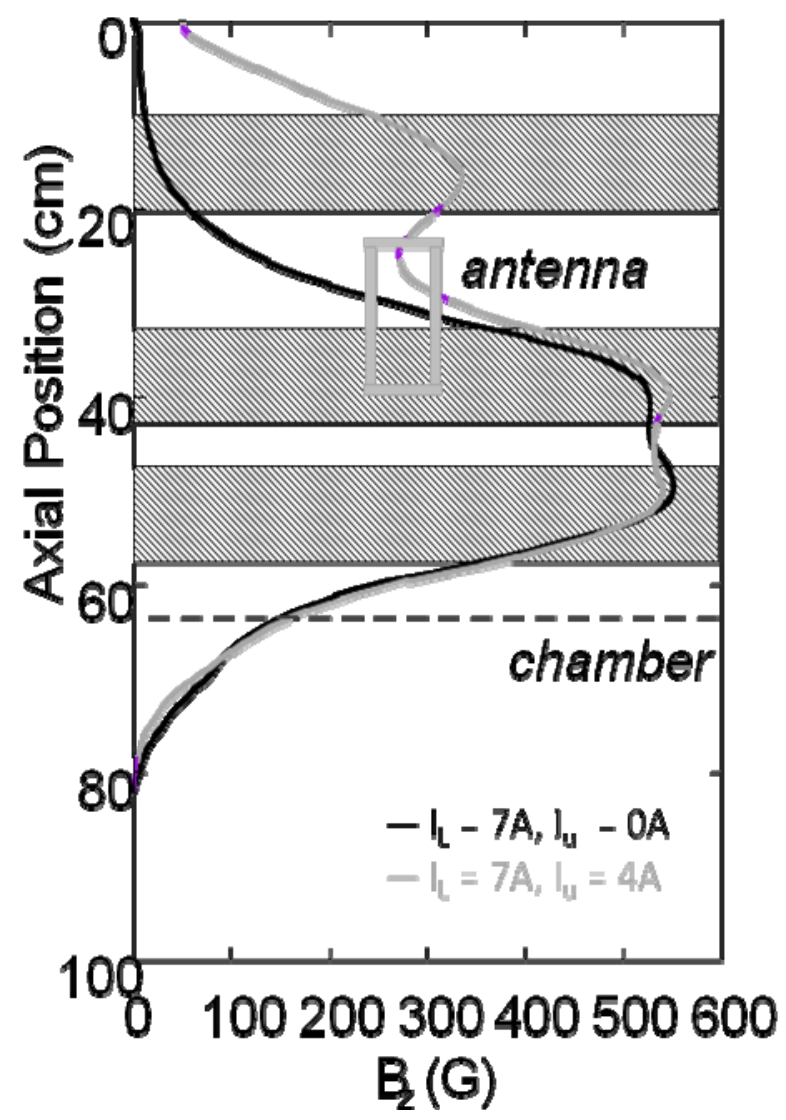

Figure 9 CHEWIE magnetic field profile

The magnetic field strength ranged from $0-350$ Gauss for magnet 1 . The magnetic field strength for magnet 2 and magnet 3 ranged from $0-550$ Gauss. The strongest part of the magnetic field was between magnet 2 and magnet 3 . The magnetic field appeared relatively uniform over this region. At $63.5 \mathrm{~cm}$, the expansion region began and the magnetic field rapidly decreased from 125 Gauss to 0 Gauss. Table 1 shows the conversion between the current in two power supplies to the magnetic field strength at the magnets. 
Table 1 The table shows the conversion from the current through the power supply to the magnetic field strength of the three magnets.

\begin{tabular}{|c|c|c|c|}
\hline $\begin{array}{l}\text { Current in magnet } 1 \\
\text { (A) }\end{array}$ & $\begin{array}{l}\text { B } \\
\text { (Gauss) }\end{array}$ & Current in magnet 2 and magnet $3(\mathrm{~A})$ & $\begin{array}{l}\text { B } \\
\text { (Gauss) }\end{array}$ \\
\hline 0 & 0 & 0 & 0 \\
\hline 1 & 87.5 & 1 & 78.6 \\
\hline 2 & 175 & 2 & 157.2 \\
\hline 3 & 262.5 & 3 & 235.8 \\
\hline 4 & 350 & 4 & 314.4 \\
\hline & & 5 & 393 \\
\hline & & 6 & 471.6 \\
\hline & & 6.5 & 510.9 \\
\hline & & 7 & 550.2 \\
\hline
\end{tabular}

Pressure measurements were taken with a Kurt J. Lesker 4500 ion gauge controller which used a thermocouple and an ion gauge to measure the pressure. The thermocouple read the pressure from 1 Torr to 1 mTorr. The ion gauge determined the pressure less than 1 mTorr to $10^{-10}$ Torr. The filament in the ion gauge could not operate at pressures greater than 1 mTorr [Kurt J. Lesker Company].

Gas flow was regulated by an MKS PR4000 power supply and an MKS 1179A mass-flow controller. The lowest achieved flow rate was 13 sccm (standard cubic centimeters per minute), which corresponded to a pressure of 2 mTorr. This limitation was due to the size of the aperture of the mass flow controller. A new mass flow controller with a smaller aperture would need to be purchased in order to achieve lower pressures. A TMU 521P turbomolecular drag pump replaced the original diffusion pump. The diffusion pump would generate a back flow of oil which would travel into the expansion chamber and contaminate the plasma. The turbomolecular pump was backed by an XtraDry 150-2 Dry piston roughing pump. 
A $20 \mathrm{MHz}$ Agilent 33220A function generator provided an RF frequency of 12.8 MHz and $15 \mathrm{MHz}$ to the plasma antenna. The output signal was amplified by two different amplifiers, a $50 \mathrm{~W}$ Wide Band RF amplifier - Linear Model FK30-50 and the Henry Radio 2 KP Classic Linear Amplifier. First, the Linear Model FK30-50 amplified the output from the function generator. Then, the Henry Radio amplified the output from the first amplifier and drove the double saddle antenna. Arcing occurred in the Henry Radio Amplifier when the function generator provided too much voltage. A Bird Electronic Corporation Wattmeter was used to measure the forward and reverse power before reaching the $\Pi$ type matching network. The forward power was sent from the amplified output signal. The reverse power was the power reflected from the antenna. The Henry Radio had a tuning capacitor and a tunable load inductor. А П type matching network was placed between the antenna and Henry Radio amplifier. In the $\Pi$ type matching network, the antenna inductance was matched by tuning and load capacitors. 


\section{Experimental Results}

The Langmuir probe experiments were performed in the inductive mode. The four experiments were to measure the plasma position, forward power, pressure, and magnetic field strength to determine the electron temperature and electron density with the Langmuir probe. The uncertainty in the Langmuir probe measurements were two significant figures. The LIF experiments were performed in the helicon mode at low pressure. For the high pressure cases, the LIF experiments were performed in the inductive mode. From the Langmuir probe traces, some of the parameters, such as the Debye length of the plasma and the mean free path of the particles, could be determined. From the LIF experiments, ion velocity distribution functions were determined, which were used to calculate bulk ion velocity and ion temperature. The Debye length was $0.000055 \mathrm{~m}$ at $12.8 \mathrm{MHz}$, and $0.000036 \mathrm{~m}$ at $15 \mathrm{MHz}$. At 550 Gauss, the electron cyclotron frequency was $9.710^{9} \mathrm{~Hz}$, while the ion cyclotron frequency was $1.310^{5} \mathrm{~Hz}$. The helicon wave propagated at around $10^{7} \mathrm{~Hz}$. The electron plasma frequency at 12.8 $\mathrm{MHz}$ was $210^{7} \mathrm{~Hz}$, and $410^{7} \mathrm{~Hz}$ at $15 \mathrm{MHz}$.

\subsection{Langmuir Probe Measurements}

The possible cause for uncertainty in electron temperature measurements is the error in the collected current. The uncertainty of the electron density depends on the error in the electron temperature, space potential, current collected, and the area of the probe.

The first experiment, as shown in Figure 10, determined the electron temperature and electron density as a function of position in the plasma. The Langmuir probe started in the center of the chamber which corresponded to a radial distance of 0 . The probe tip 
was then moved in $0.5 \pm 0.1 \mathrm{~cm}$ increments to $5 \pm 0.1 \mathrm{~cm}$. The experimental conditions were an RF frequency of $15 \mathrm{MHz}$, RF power of 400 Watts, a pressure of $1.2 \mathrm{mTorr}$, and a magnetic field of 511 Gauss at magnet 2 and magnet 3, and 0 Gauss for magnet 1 . The electron temperature was hotter in the center at $12 \mathrm{eV}$ and cooler towards the walls at $9 \mathrm{eV}$. The electron density was uniform over a radial distance of $4.0 \pm 0.1 \mathrm{~cm}$ at $510^{11} \mathrm{~cm}^{-3}$.

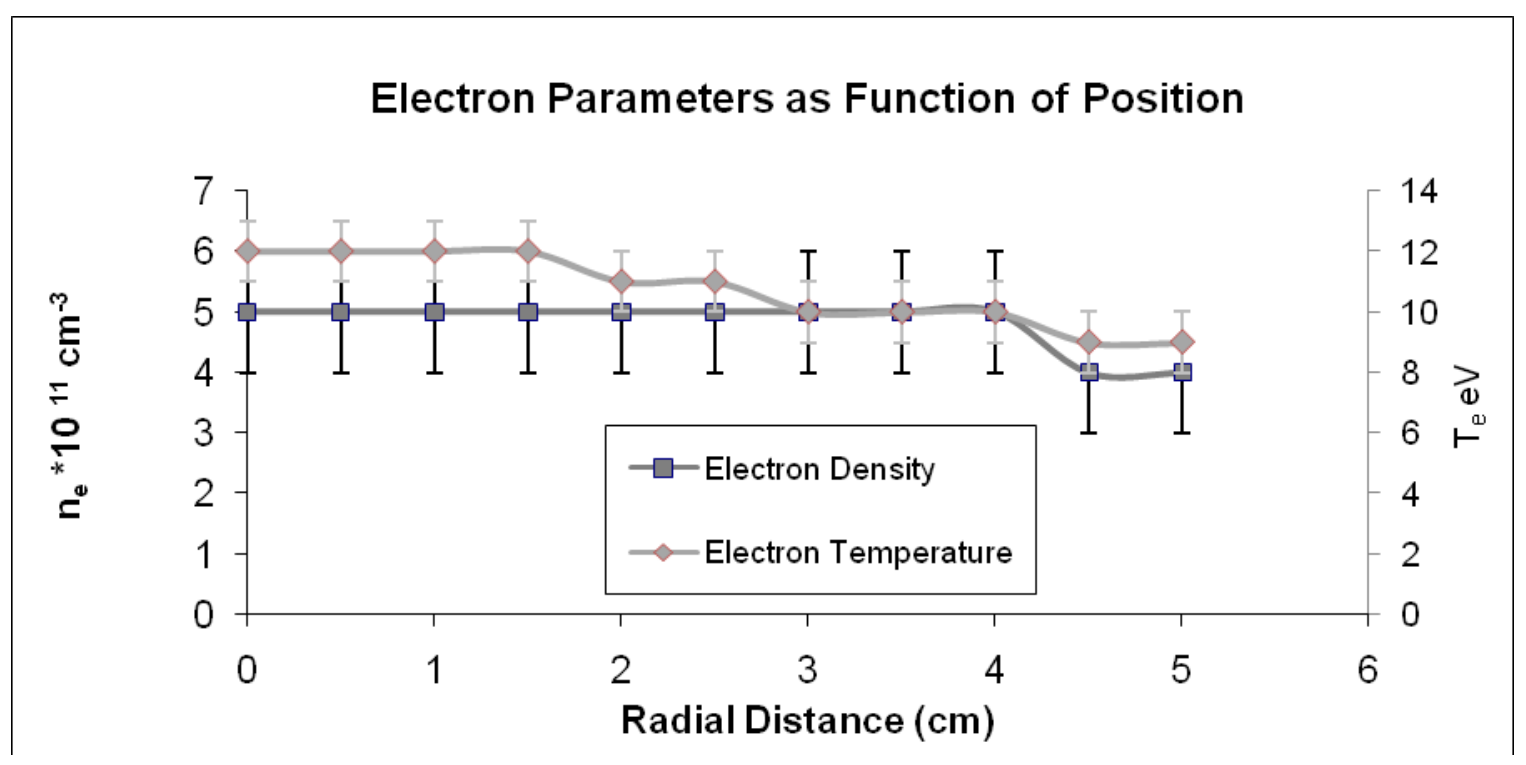

Figure 10 Electron density and electron temperature as a function of position. The conditions for the experiment were a pressure of $1.2 \mathrm{mTorr}$, an RF frequency $15 \mathrm{MHz}$, and an RF power of $400 \mathrm{~W}$. Magnet 2 and magnet 3 were at 6.5 amps, and magnet 1 was at 0 Amps.

The second experiment, as shown in Figure 11, measured the electron temperature and electron density as a function of forward power, as measured with a Bird meter. A slight hysteresis was observed when increasing the function generators voltage by $100 \mathrm{mV}$ from $0 \mathrm{mV}$ to $1100 \mathrm{mV}$ and then decreasing the voltage from $1100 \mathrm{mV}$ to $0 \mathrm{mV}$ by $100 \mathrm{mV}$. The data showed two curves which corresponded to increasing the power and then decreasing the power. In this experiment, the RF frequency was $12.8 \mathrm{MHz}$, a 
flow rate of $30 \mathrm{sccm}$, and a pressure of 3.8 mTorr. Near magnet 1, the magnetic field was 350 Gauss, while the magnetic field was 550 Gauss near magnet 2 and magnet 3 . The measurement position was the center of the chamber. The electron density saturated at $910^{10} \mathrm{~cm}^{-3}$ when the forward power exceeded 250 Watts. The electron temperature decreased with power until the forward power reached 150 Watts. Any further increase in the power left the electron temperature constant at $5 \mathrm{eV}$. A hysteresis effect was not observed. However, if a hysteresis was present, the independent variable would yield two or more dependent variables. Since a hysteresis was not present, the condition of the new state was not dependent upon the condition of the old state which means the electron temperature and electron density depends on the current forward power. This result made taking measurements easier since the forward case and reverse case yield the same value.

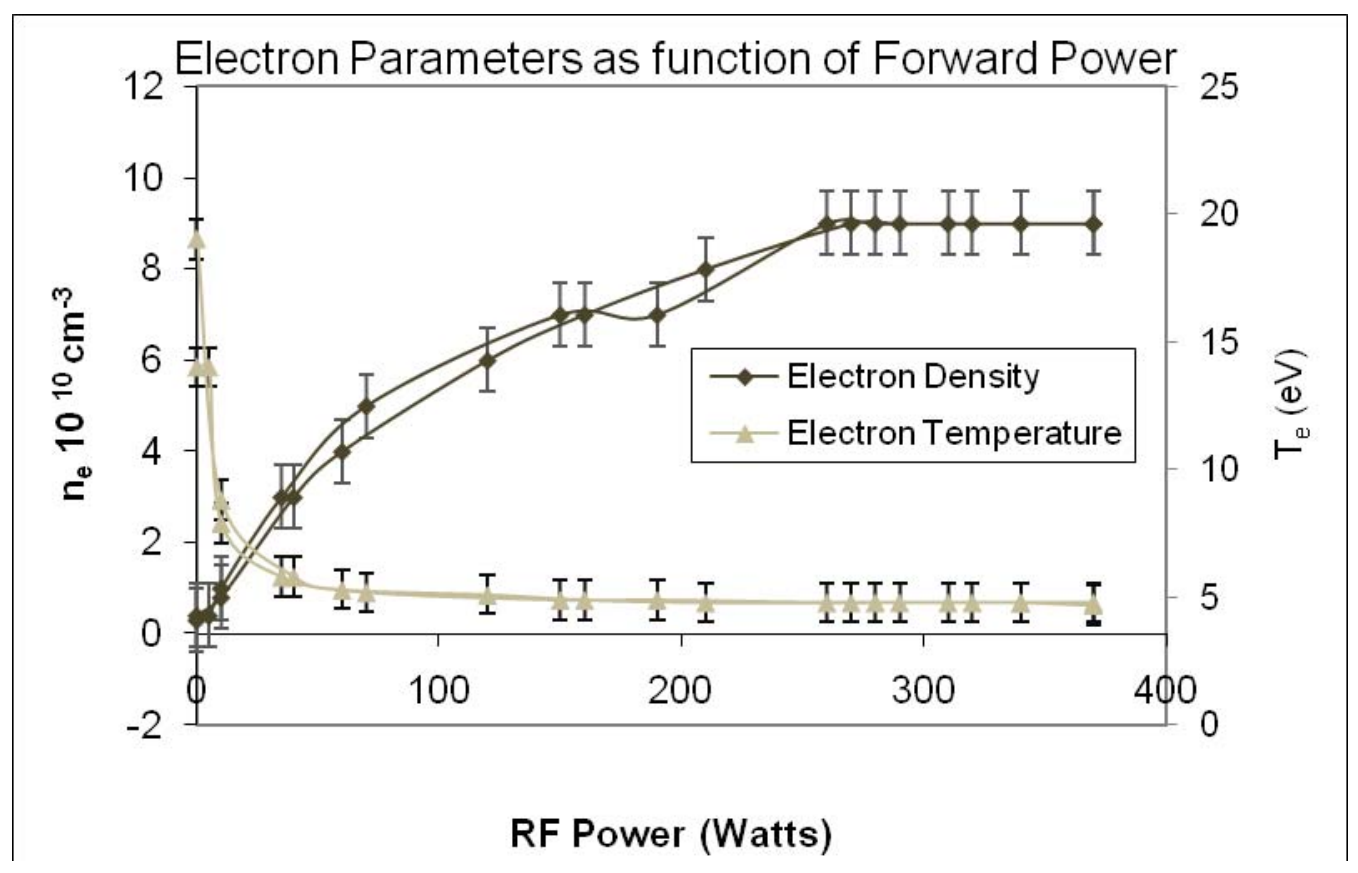

Figure 11 Electron density and electron temperature as a function of RF power. Magnet 1 was 4 amps while magnet 2 and magnet 3 were at 7 amps. The pressure was at 3.8 mTorr and the flow rate was $30 \mathrm{sccm}$. The RF frequency was $12.8 \mathrm{MHz}$. As the RF power increases, the electron density saturated. 
The third experiment, as shown in Figure 12, measured the dependence of electron density and electron temperature on the fill pressure. The pressure was adjusted by increasing the gas flow rate in $10 \mathrm{sccm}$ increments starting at $20 \mathrm{sccm}$ and reaching $150 \mathrm{sccm}$. Then, the pressure was decreased by $10 \mathrm{sccm}$ from $150 \mathrm{sccm}$ to $20 \mathrm{sccm}$. This resulted in the pressure increasing from $3 \mathrm{mTorr}$ to $12 \mathrm{mTorr}$ and then decreasing from 12 mTorr to 3 mTorr. This was to measure any hysteresis in the plasma response. The conditions for the experiment were an RF frequency of $12.8 \mathrm{MHz}$ and an RF power of 240 Watts. The magnetic field was 350 Gauss at magnet 1 . Magnet 2 and Magnet 3 had a magnetic field strength of 550 Gauss. The electron temperature remained constant at $5 \mathrm{eV}$ from a pressure of $3-12 \mathrm{mTorr}$. The electron density was approximately $110^{11} \mathrm{~cm}^{-3}$ from 3 - 12 mTorr. Changing the pressure affects ionizing collisions between particles. A noticeable hysteresis effect was not observed. This means that the electron density and electron temperature were only dependent on the current pressure. 


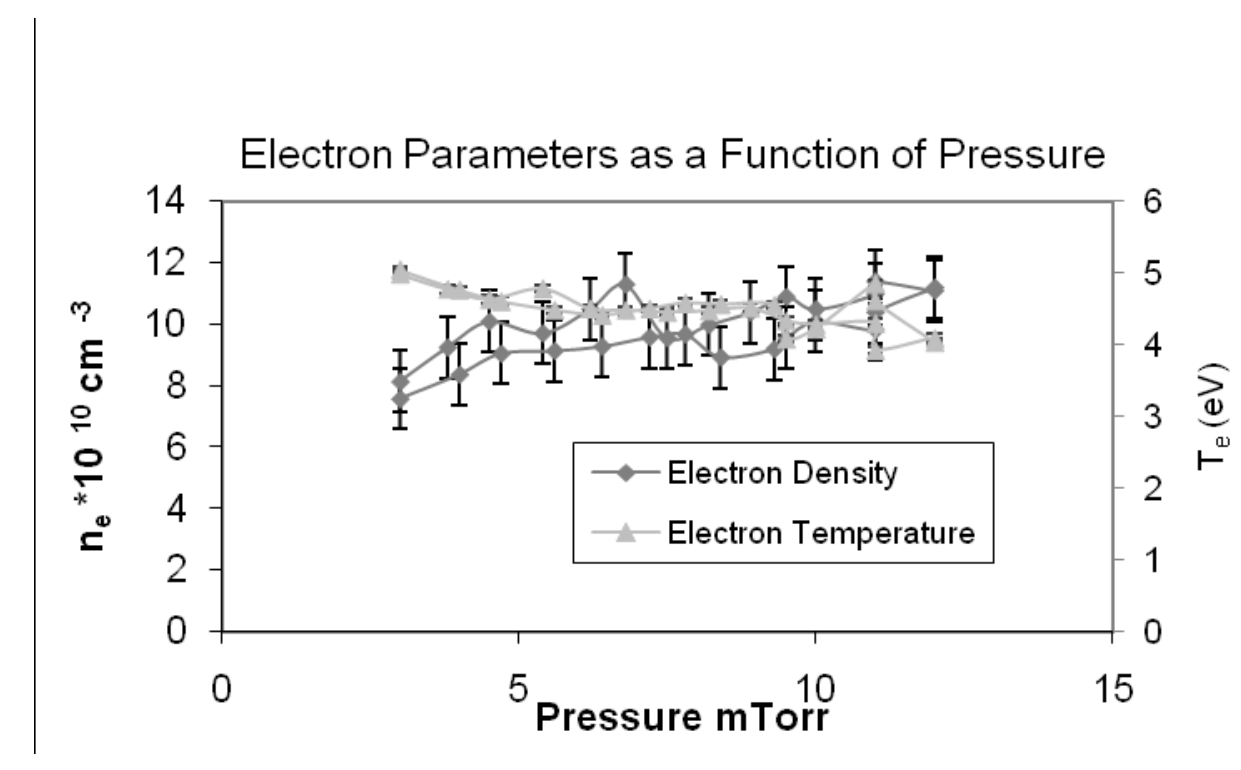

Figure 12 Electron density and electron temperature as a function of fill pressure. The current through magnet 1 was 4 amps and the current through the magnet 2 and magnet 3 were 7 amps. The forward power was $240 \mathrm{~W}$ Forward and the RF frequency was $12.8 \mathrm{MHz}$. The flow rate was increased from $20 \mathrm{sccm}$ to $150 \mathrm{sccm}$ which resulted in an increase of pressure. Then, the flow rate was decreased from $150 \mathrm{sccm}$ to $20 \mathrm{sccm}$ which resulted in a decrease of pressure.

The fourth experiment, as shown in Figures 13 and 14, determined the electron density and electron temperature dependence upon the magnetic field strength. The experimental conditions were $12.8 \mathrm{MHz}$, a gas flow rate of $30 \mathrm{sccm}$, a pressure of $3.8 \mathrm{mTorr}$, and a forward power of 400 Watts. The current through magnet 1 varied from 1 to 4 amps which meant the magnetic field changed from 87.5 Gauss to 350 Gauss. The current through magnet 2 and magnet 3 were varied from 0 to 7 amps, which corresponded to a change in the magnetic field of 0 Gauss to 550 Gauss. When the magnet field strength at magnet 1 was 262.5 or 350 Gauss and the magnetic field strength at magnet 2 and 3 increased from 0 to 550 Gauss, the electron density increased from $810^{10} \mathrm{~cm}^{-3}$ to $110^{11} \mathrm{~cm}^{-3}$. When the magnetic field strength at magnet 1 was 87.5 or 175 Gauss, and the magnetic field at magnet 2 and magnet 3 increased from 0 Gauss to 393 Gauss, the electron density increased from $810^{10} \mathrm{~cm}^{-3}$ to $110^{11} \mathrm{~cm}^{-3}$. As the 
magnetic field through magnet 2 and magnet 3 increased from 393 Gauss to 550 Gauss, the electron density decreased. The general pattern was that the electron density increased with magnetic field strength. The electron temperature ranged from 4.5 to $5 \mathrm{eV}$. When the magnetic field strength through magnet 1 remained fixed, the electron temperature appeared relatively constant. When the magnetic field strength increased through magnet 1 , the electron temperature decreased. At larger magnetic fields, the gyro-radius of an electron was smaller which caused more particles to be trapped in the plasma.

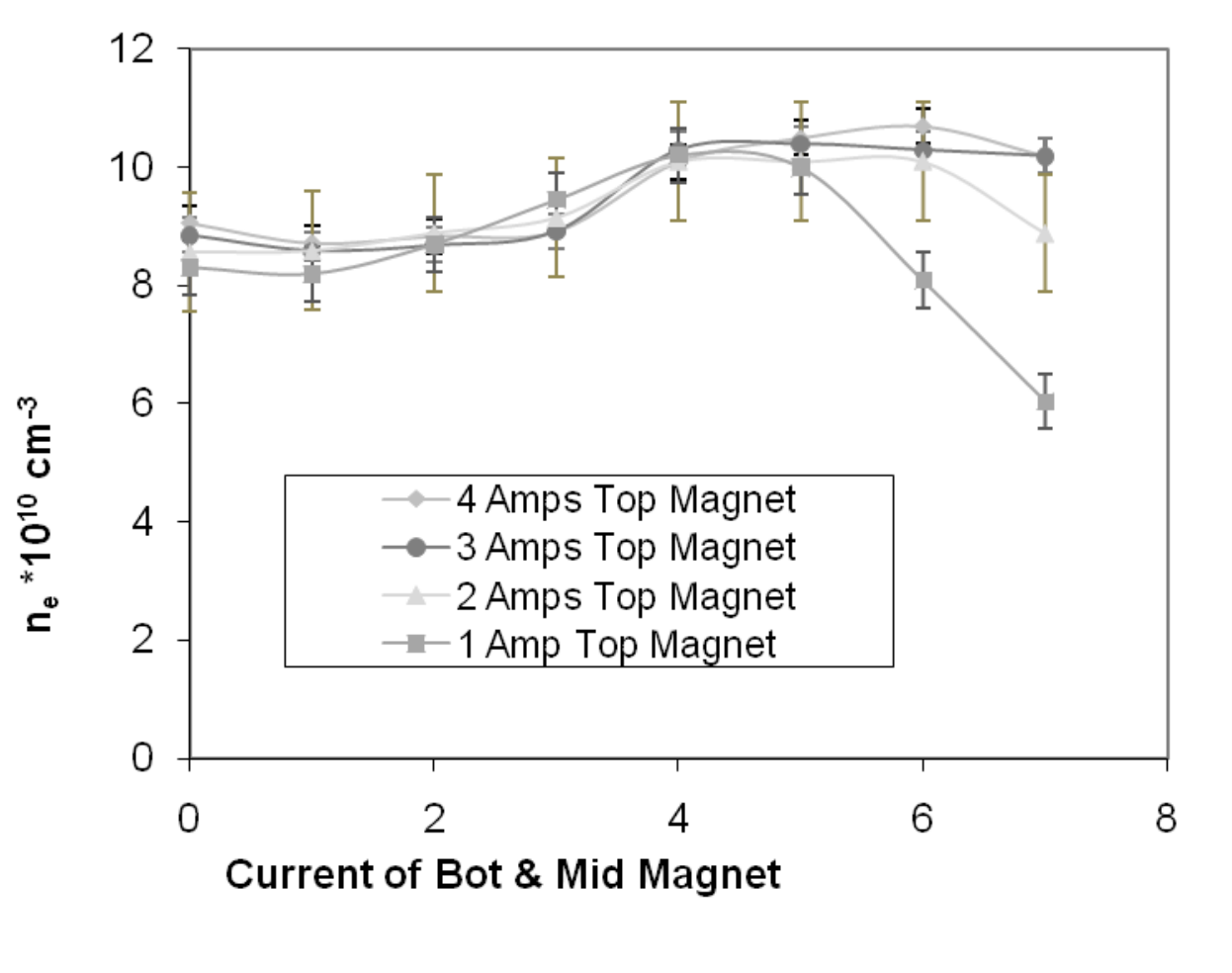

Figure 13 Electron density as a function of electromagnet current. The flow rate was $30 \mathrm{sccm}$, the pressure was $3.8 \mathrm{mTorr}$. The forward power was $400 \mathrm{~W}$ and the RF frequency was $12.8 \mathrm{MHz}$. The electron density increased with magnetic field strength. 


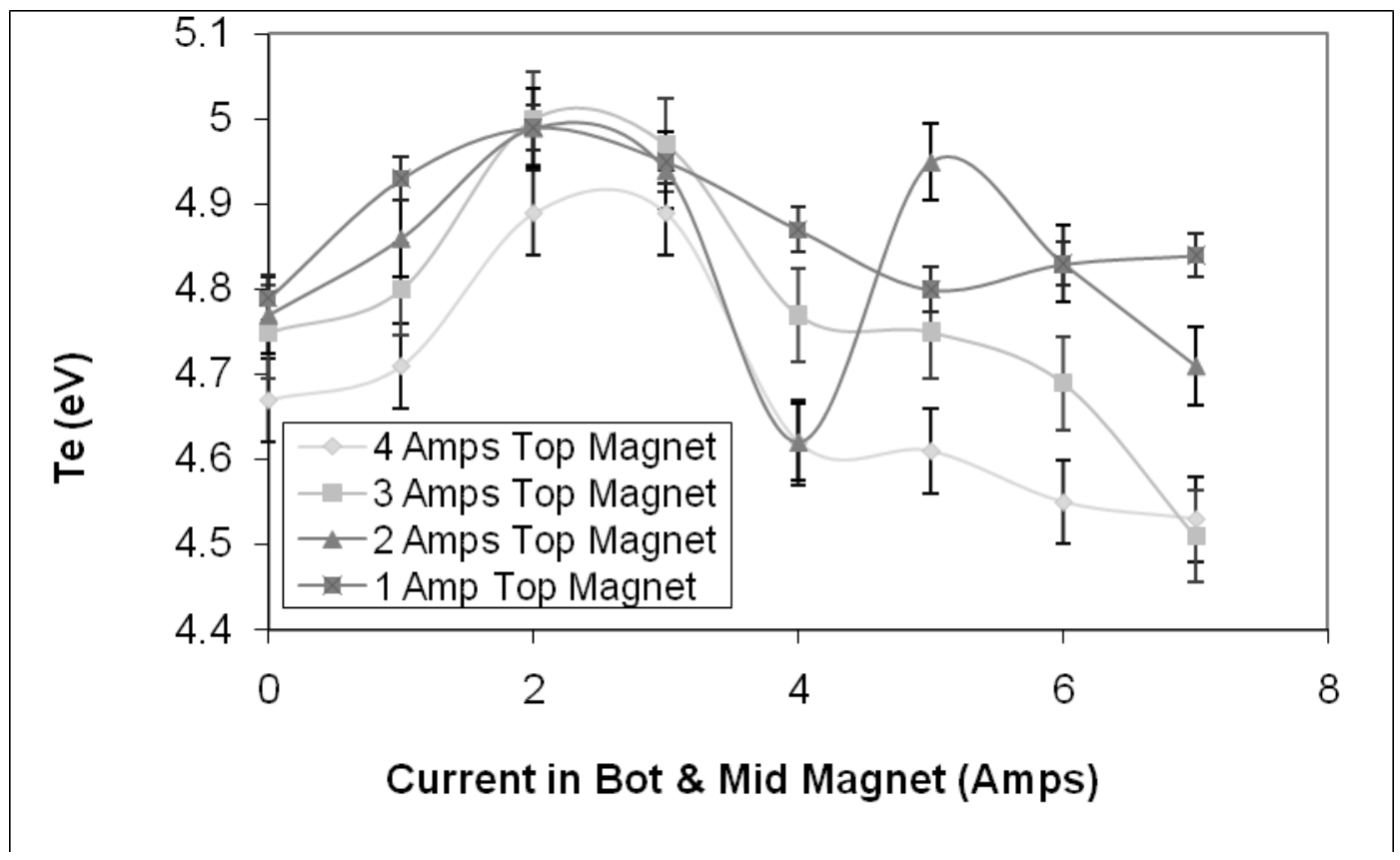

Figure 14 Electron temperature as a function of electromagnet current. The flow rate was $30 \mathrm{sccm}$, and was a pressure of $3.8 \mathrm{mTorr}$. The RF frequency was $12.8 \mathrm{MHz}$ and the forward power was $400 \mathrm{~W}$. 
Langmuir probe trace, as shown in Figure 15, showed the electrostatic instability at high magnetic field strengths. The current through magnet 2 and magnet 3 was 7 amps and its magnetic field was 550 Gauss. As the bias voltage increased, the size of the oscillation also grew. The cause for the instability at high magnetic fields was not known.

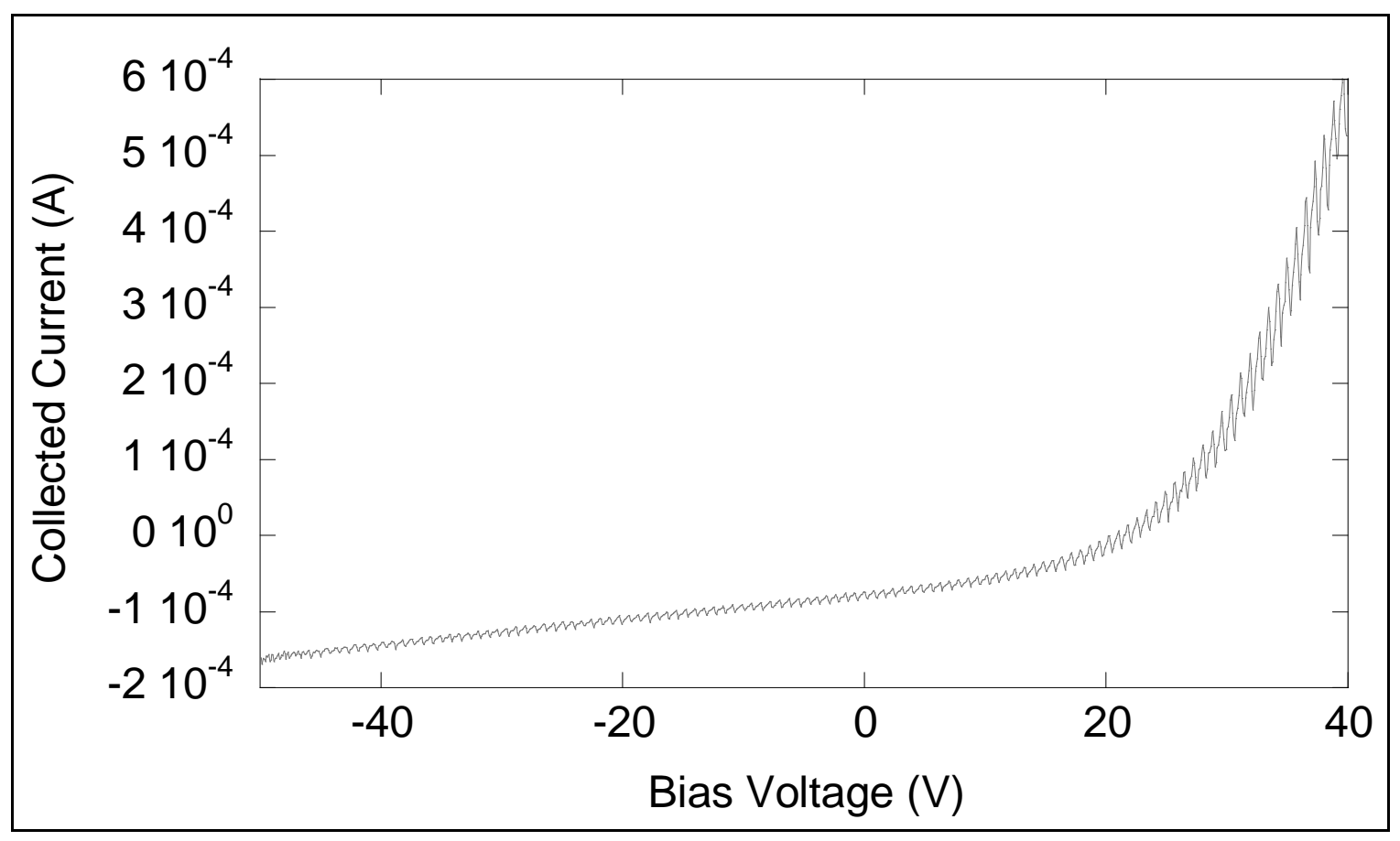

Figure 15 Langmuir probe I-V curve for maximum current in magnet 2 and magnet 3

\subsection{Laser Induced Fluorescence Measurements}

The parallel ion velocity distribution functions were measured at different gas pressures. An ion beam moved away from the laser beam and was observed at a frequency lower than its rest frequency. Therefore, the slow population centered at a lower frequency and the ion beam centered at a higher frequency. The Full Width Half Maximum (FWHM) of the spectral line determined the ion temperature of the plasma, while the frequency shift provided the ion velocity. The ion Bohm velocity or ion 
acoustic velocity entering the sheath was $3500 \mathrm{~m} / \mathrm{s}$ for an RF frequency of $12.8 \mathrm{MHz}$ and $5400 \mathrm{~m} / \mathrm{s}$ at an RF frequency of $15 \mathrm{MHz}$. Using the velocity of the beam and the ion acoustic velocity it was possible to calculate the Mach number of the beam where $M a=v_{0} / c_{s}$. The ion acoustic velocity equaled the speed of sound in the medium, $c_{s}$, and $v_{0}$ was the velocity of the ions relative to the lab frame. For the ion temperature, there was uncertainty in the FWHM of the LIF intensity, and the laser wavelength. In dealing with ion velocity, there was uncertainty in the frequency shift between the two Gaussians and the laser wavelength. The uncertainty in the frequency of the laser was small compared to the uncertainty of the FWHM. The uncertainty in the temperature was determined by

$$
\delta T / T=n \delta \omega_{D} / \omega_{D}
$$

where $n=2$. The uncertainty in velocity was calculated as

$$
\delta v_{i t h} / v_{i t h}=\delta \Delta v / \Delta v
$$

In the first experiment, as shown in Figure 16, the conditions were an RF frequency of $15 \mathrm{MHz}$, an RF power of 250 Watts, a gas pressure of 2.5 mTorr, and 6.5 amps through magnet 2 and magnet 3. The magnetic field strength between the two magnets was 511 Gauss. The magnetic field strength at magnet 1 was 0 Gauss since the current through magnet 1 was 0 amps. The fit to the intensity of the line was a single, well defined Maxwellian. The FWHM was $3.4 \pm 0.8 \mathrm{GHz}$. Using the Doppler Broadening Parameter (equation 11) and multiplying the RHS by 1.665, the ion temperature was determined to be $0.3 \pm 0.2 \mathrm{eV}$. The Doppler shift of $0.5 \pm 0.1 \mathrm{GHz}$ between the iodine cell and the LIF signal, gave a velocity of the ion population of $400 \pm$ $100 \mathrm{~m} / \mathrm{s}$ using Eq (13). 


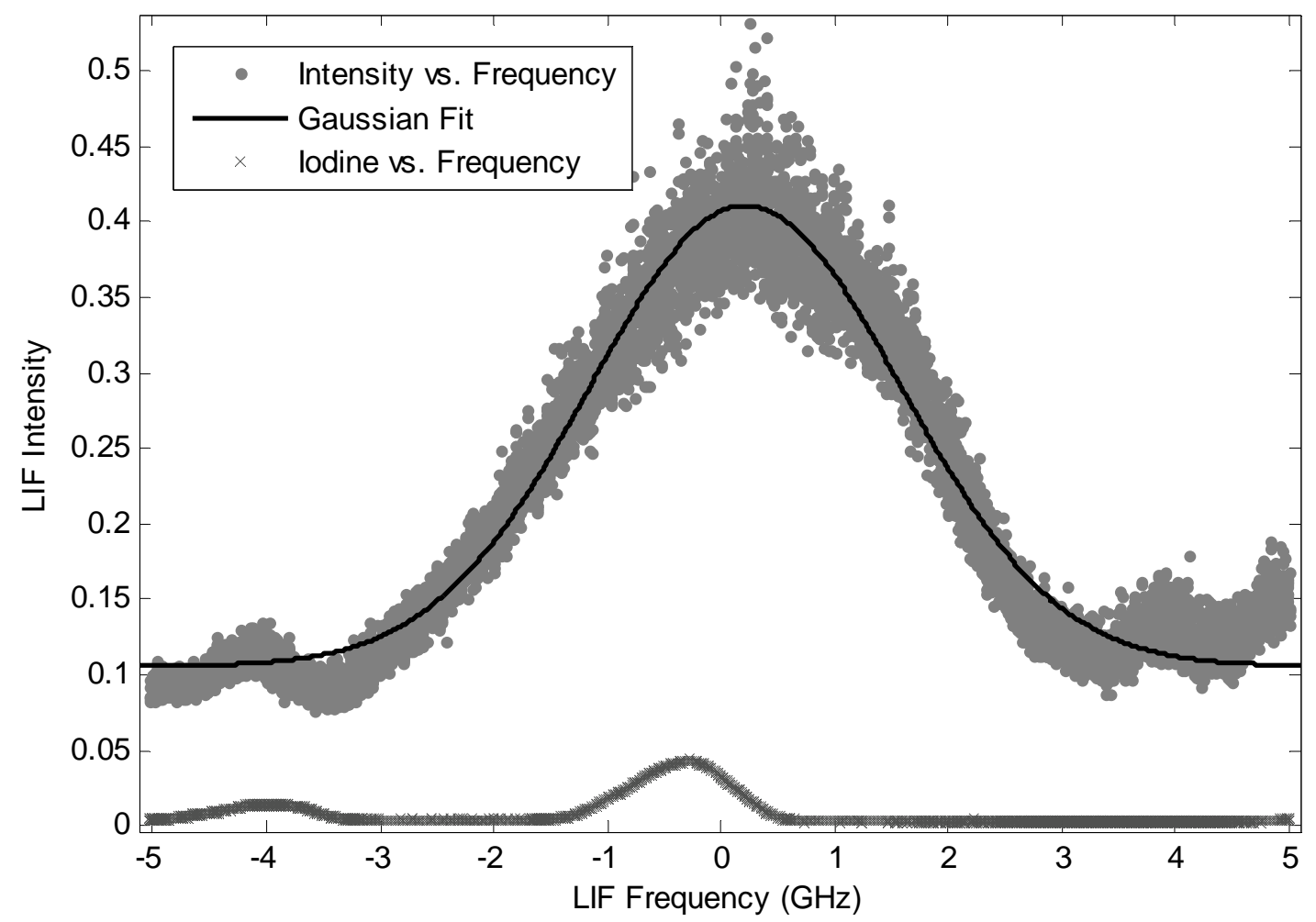

Figure 16 LIF intensity versus laser frequency at a pressure of $2.5 \mathrm{mTorr}$. This provided the iodine cell signal, LIF intensity, and a Gaussian fit for the LIF signal. The FWHM was $3.4 \pm 0.8 \mathrm{GHz}$ and ion temperature was $0.3 \pm 0.2 \mathrm{eV}$. The equation is $\mathrm{y}=0.3051 * \exp \left(-((\mathrm{x}-0.2165) / 1.938)^{2}\right)+0.1058$

In the second experiment, as shown in Figure 17, the pressure was changed to 1.2 mTorr and a forward power of 250 Watts. The LIF signal was fit to two Gaussians. The FWHM of the first Gaussian was approximately $4.3 \pm 1.1 \mathrm{GHz}$. The ion temperature was determined to be $0.5 \pm 0.3 \mathrm{eV}$ by using Eq (12). The ion population appeared to be shifted by $4.0 \pm 0.1 \mathrm{GHZ}$. Using Eq (13), the calculated ion velocity was $2900 \pm 100$ $\mathrm{m} / \mathrm{s}$. The Mach number of the beam was 0.54 . 
LIF Data 10/22/2007

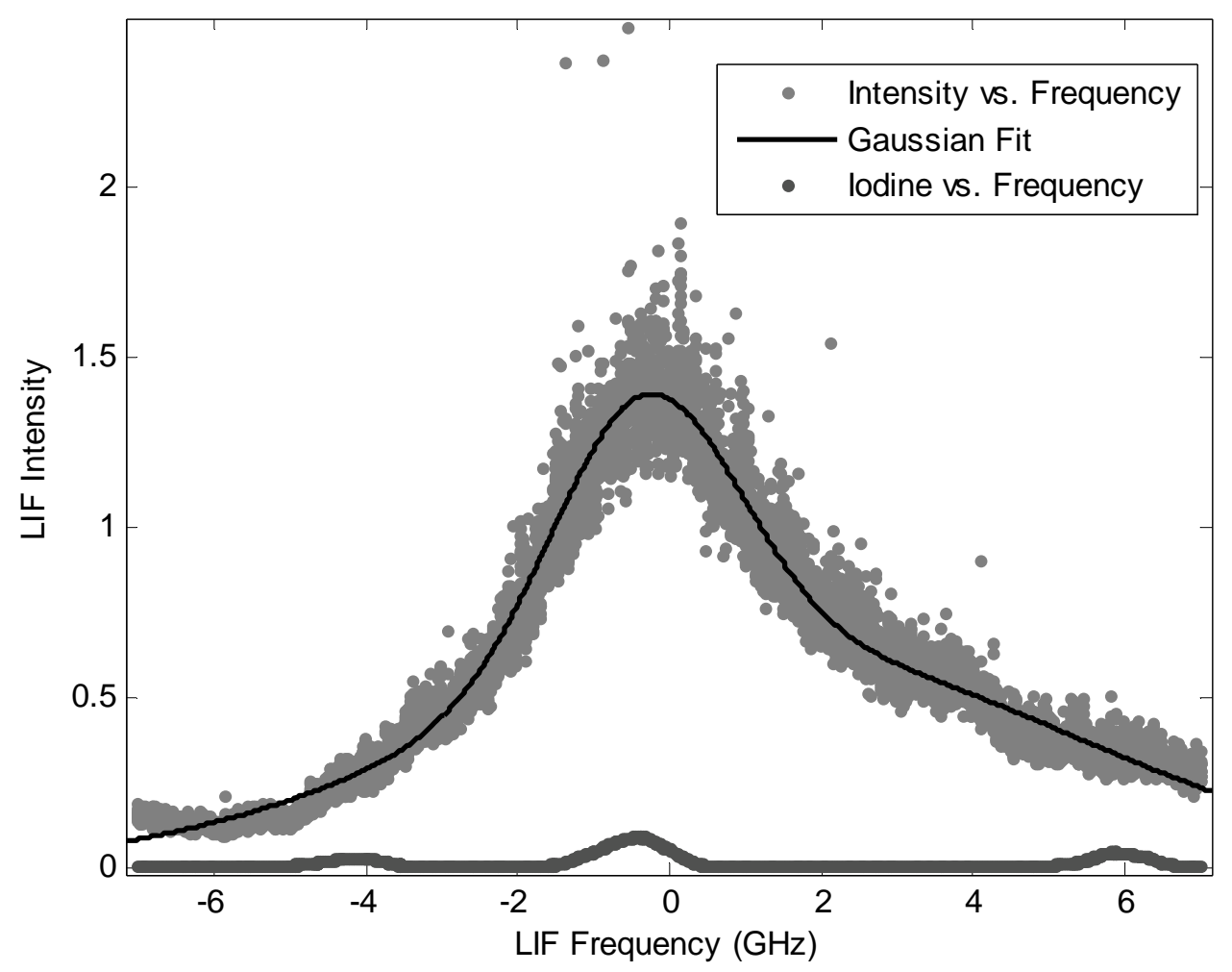

Figure 17 LIF intensity versus laser frequency at a pressure of $1.2 \mathrm{mTorr}$. The FWHM was 4.3 $\pm 1.1 \mathrm{GHz}$ and the ion temperature was $0.5 \pm 0.3 \mathrm{eV}$. The equation to fit the LIF trace is $y=0.7987 * \exp \left(-((x+.3267) / 1.722)^{2}\right)+0.6368 * \exp \left(-((x-1.241) / 5.775)^{2}\right)$

In the third experiment, as shown in Figure 18, the pressure was 1.2 mTorr, an RF power of 360 Watts, and the FWHM was $4 \pm 0.8 \mathrm{GHZ}$. This meant that the calculated ion temperature was $0.5 \pm 0.2 \mathrm{eV}$. The shifted frequency was $4.0 \pm 0.1 \mathrm{GHz}$, which confirmed that the calculated ion velocity was $2900 \pm 100 \mathrm{~m} / \mathrm{s}$. Again, the Mach number was 0.54 . 


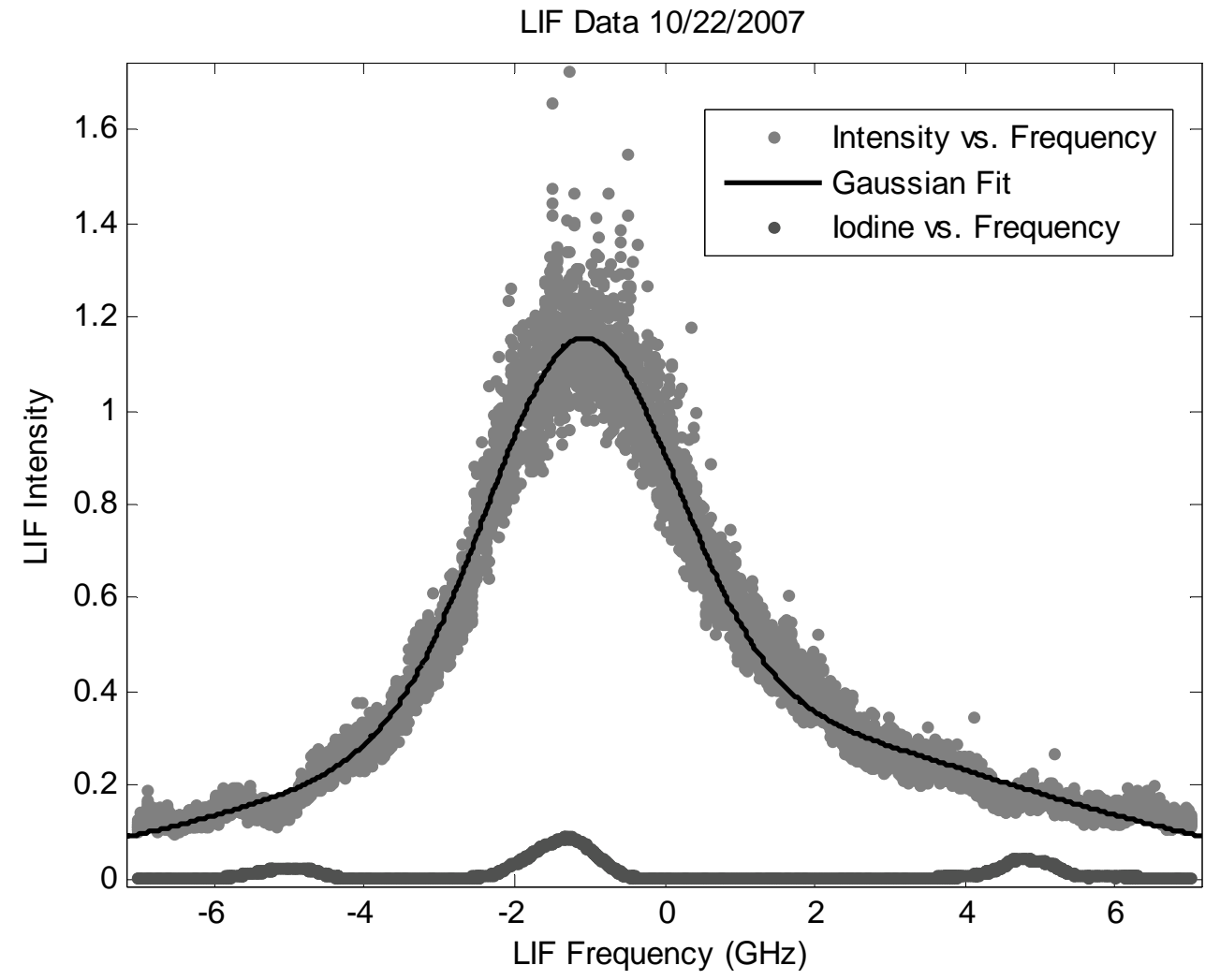

Figure 18 LIF intensity versus laser frequency at a pressure of $1.2 \mathrm{mTorr}$. The FWHM was $4.0 \pm 0.1 \mathrm{GHz}$ and ion temperature was $0.5 \pm 0.2 \mathrm{eV}$. The equation describing the fit is $y=0.8118 * \exp \left(-((x+1.1) / 1.774)^{2}\right)+0.3552 * \exp \left(-((x-0.021463) / 6.141)^{2}\right)$ 


\section{Conclusion}

These experiments measured the electron temperature and electron density in the inductively coupled mode for the CHEWIE apparatus with a Langmuir probe. The ion velocity and ion temperature were determined in the helicon mode with LIF for low pressure. At high pressure, LIF was performed in the inductive mode.

The first experiment measured the electron density and electron temperature as a function of plasma position. The RF frequency was $15 \mathrm{MHz}$, while the other experiments were performed at $12.8 \mathrm{MHz}$. In this experiment, the RF power was $400 \mathrm{Watts}$, the pressure was 1.2 mTorr, and the magnetic field was 511 Gauss for magnet 2 and magnet 3 and 0 Gauss for magnet 1 . The electron temperature was larger in the center of the chamber at $12 \mathrm{eV}$ and cooler towards the walls at $9 \mathrm{eV}$. The electron density was uniform over a radial distance of $4 \mathrm{~cm}$.

In the second Langmuir probe experiment, the RF power was varied to measure the electron temperature and electron density. The experimental conditions were an RF frequency of $12.8 \mathrm{MHz}$ and a neutral pressure of 3.8 mTorr. The magnetic field for magnet 1 was 350 Gauss and the magnetic field for magnet 2 and magnet 3 was 550 Gauss. The electron density saturated at a value of $110^{11} \mathrm{~cm}^{-3}$ after achieving a forward power of 250 Watts. Above 150 Watts of forward power, the electron temperature remained constant at $5 \mathrm{eV}$. The strength of the RF power was proportional to the plasma density until saturation was reached. The electron temperature was larger at low RF power since the plasma was weakly ionized. Hysteresis was not observed where the electron temperature and electron density was dependent only on the current state of the forward RF power. 
The third experiment measured the electron temperature and electron density dependence on neutral gas pressure. The measurements were performed at an RF frequency of $12.8 \mathrm{MHz}$, a forward power of 240 Watts, and a magnetic field of 350 Gauss at magnet 1 and 550 Gauss at magnet 2 and magnet 3. The electron temperature and electron density remained constant at $5 \mathrm{eV}$ and $110^{11} \mathrm{~cm}^{-3}$, respectively, as the pressure ranged from 3 to 12 mTorr. Again, a hysteresis was not observed. Thus, the electron temperature and electron density depended on the current pressure of the system.

The fourth experiment measured the electron temperature and electron density as a function of magnetic field profile and strength. The experimental parameters were an RF frequency of $12.8 \mathrm{MHz}$, a forward power of 400 Watts, and a neutral gas pressure of 3.8 mTorr. The largest electron density, $110^{11} \mathrm{~cm}^{-3}$, was observed at 350 Gauss at magnet 1 and 550 Gauss at magnet 2 and magnet 3. The maximum electron temperature of $5 \mathrm{eV}$ was at 157 Gauss for magnet 2 and magnet 3. At 550 Gauss through magnet 2 and magnet 3, an electron temperature of $4.5 \mathrm{eV}$ was observed. A larger magnetic field led to a smaller gyro-radius and allowed for electrons and ions to stay trapped in the plasma, which led to a larger plasma density. Changing the gyro-radius did not have a substantial effect on the electron temperature.

By comparing the first experiment to the last three experiments, it was possible to observe frequency dependence on electron temperature and electron density. At an RF frequency of $12.8 \mathrm{MHz}$ and measurements taken at the center of the chamber, the electron density was $110^{11} \mathrm{~cm}^{-3}$ and the electron temperature was $5 \mathrm{eV}$. When the experiment was performed at the center of the chamber with an RF frequency of $15 \mathrm{MHz}$, 
the electron density was $510^{11} \mathrm{~cm}^{-3}$ and the electron temperature was $12 \mathrm{eV}$. The electron temperature and electron density increased with RF frequency. It may be desirable to determine how the electron temperature and electron density change with RF frequency.

For the LIF experiments, the ion velocity distribution functions were measured as a function of pressure. The high pressure experiment was performed at 2.5 mTorr, a magnetic field of 511 Gauss through magnet 2 and magnet 3 and 0 Gauss through magnet 1. A single, Maxwellian distribution was observed with a FWHM maximum was 3.4 $\pm 0.8 \mathrm{GHz}$. The frequency shift between the iodine cell and Maxwellian was $0.5 \pm 0.1 \mathrm{GHz}$. The ion temperature was $0.3 \pm 0.2 \mathrm{eV}$ and an ion moved at $400 \pm 100 \mathrm{~m} / \mathrm{s}$. The velocity was relatively small and would not constitute a beam. This means a double layer was not present at high pressures. At high pressures, more collisions occurred between particles, such as ion-neutral and ion-electron collisions. If too many collisions occurred, ions lost energy and thus had a smaller bulk velocity.

The low pressure experiment was performed at 1.2 mTorr and a magnetic field of 511 Gauss between magnet 2 and magnet 3 and 0 Gauss at magnet 1. Two Gaussians were observed in the low pressure cases. For the first case, the FWHM was $4.3 \pm 1.1 \mathrm{GHz}$, which meant the ion temperature was $0.5 \pm 0.3 \mathrm{eV}$. The frequency shift between the first Maxwellian and the iodine cell was $0 \pm 0.1 \mathrm{GHz}$, which corresponded to the rest velocity. The frequency shift between the two Maxwellians was $4.0 \pm 0.1 \mathrm{GHz}$, which corresponded to an ion velocity of $2900 \pm 100 \mathrm{~m} / \mathrm{s}$. The second low pressure case had a FWHM of $4.0 \pm 0.8 \mathrm{GHZ}$ and an ion temperature of $0.5 \pm 0.2 \mathrm{eV}$. The frequency shift between the first Maxwellian and the iodine cell was $0.0 \pm 0.1 \mathrm{GHz}$ and the ions 
were at rest. Again, the frequency shift was $4.0 \pm 0.1 \mathrm{GHz}$ and the ion velocity was $2900 \pm 100 \mathrm{~m} / \mathrm{s}$. This suggested that an ion population was accelerated at low pressures, but not a double layer. Since fewer collisions occurred at low pressures, the ion did not lose energy which allowed a beam to form. A double layer would have larger ion velocities.

For future LIF experiments, it may be desirable to scan the ion velocity distribution functions for rarified plasmas over a range of pressures. In order to achieve rarified plasmas, a mass flow controller with a smaller aperture needs to be purchased. To confirm the presence of a double layer, it may be desirable to also measure the electron velocity distribution functions (evdf). In utilizing the evdf and ivdf, it is possible to determine the presence of a double layer since a double layer has both free electrons and free ions. Also, by scanning the pressure, it is possible to determine under what conditions a double layer forms. 


\section{References}

Bellan, P. M., Fundamentals of Plasma Physics (New York: Cambridge University, 2006).

Block, Lars P., A Double Layer Review, Astrophysics and Space Science, 55, 59-83 (1978).

Boivin, R. F., Study of the Different Line Broadening Mechanisms for the Laser Induced Fluorescence Diagnostic of the HELIX and LEIA Plasmas, PL-039 (July, 1998).

Boivin R.F. and E. E. Scime, Laser Induced Fluorescence in Ar and He Plasmas with a Tunable Diode Laser, Rev. Sci. Instrum., 74, 4352-4360 (2003).

Boswell, R. W. and F. F. Chen, Helicons - The Early Years, IEEE on Plasma Science, 25, 1229-1244 (1997).

Carlqvist, P., On the Acceleration of Energetic Cosmic Particles by Electrostatic Double Layers, IEEE Transactions on Plasma Science, 14, 794-799 (1986).

Chen, F. F. and R. W. Boswell, Helicons - The Past Decade, IEEE on Plasma Science, 25, 1245-1257 (1997).

Clarycon Co., Capacitive and Inductive Conducting, Plasma Technology for Advanced Devices, http://www.clarycon.com/plasmacoupling2.html.

Dendy, R. O., Plasma Dynamics, (USA: Oxford University Press, 1990).

Gasiorowicz, S., Quantum Physics, Third Edition (Danvers, MA: John Wiley \& Sons, Inc, 2003).

Goeckner, M.J. and J. Goree, Laser-Induced Fluorescence Measurement of Plasma Ion Temperature: Corrections for Power Saturation, J. Vac. Sci. Technol. A, 7, 977-981 (1989). 
Goertz , C. K. and G. Joyce, Numerical Simulation of the Plasma Double Layer, Astrophys. Space Sci., 32, 165-173 (1975).

Griem, H. R., Stark Broadening of Isolated Spectral Lines from Heavy Elements in a Plasma, Phys. Rev., 126, 515-523 (1962).

Greim, H. R., Spectral Line Broadening by Plasmas, (New York: Academic Press, 1974).

Griem, H. R., Priniciples of Plasma Spectroscopy, (New York: Cambridge University Press, 1997).

Hastings, D and H. Garrett, Spacecraft - Environment Interactions, (New York: Cambridge University Press, 2004).

Hecht, E., Optics, Fourth Ed, (San Fancisco: Addison Wesley, 2002).

Hill, D. N., S. Fornaca and M. G. Wickham, Single Frequency Scanning Laser as a Plasma Diagnostic, Rev. Sci. Instrum., 54, 309-314 (1983).

Kurt J. Lesker Company, KJL4500 Ionization Gauge Controller Instruction Manual.

Maurice, C., F. H. R. Feijen, and G. W. M. Kroesen, Langmuire Probe Measurements in an ICP, Department of Applied Physics, Eindhoven University of Technology, Netherlands, 2002.

Merlino, R. L., Understanding Langmuir Probe Current-Voltage Characteristics, Am. J. Phys., 75, 1078-1085 (2007).

Morton, D. C. Atomic Data for Resonance Absorption Lines I. Wavelengths Longward of the Lyman Limit, The Astrophysical Journal Supplement Series, 77, 119-202. 1991.

Quon, B. H. and A. Y. Wong, Phys. Rev. Lett. 37, 1393-1397 (1976). 
Ruzic, David N., Electric Probes for Low Temperature Plasmas, (New York: American Vacuum Society, 1994).

Salah, B. E. and M. C. Teich, Fundamentals of Photonics, (New York: John Wiley \& Sons, Inc., 1991).

Scime, E. E., P. A. Keiter, M. W. Zintl, M. Balkey, J. L. Kline, and M. E. Koepke, Control of Ion Temperature Anisotropy in a Helicon Plasma, Plasma Sources Sci. Technol. 7, 186-191 (1998).

Stenzel, R. L., Unstable Whistler-Wave Propagation along the Resonance Cone in a Large Beam-Plasma System, Phys. Rev. Lett., 38, 394-307, 1977.

Stern, R. A., Laser/Ion-Beam Diagnostics for Potential and Magnetic-Field Measurements in Plasmas (Invited), Rev. Sci. Instrum., 56, 1006-1011 (1985).

Stern, R. A. and J. A. Johnson, III, Plasma Ion Diagnostics Using Resonant Fluorescence, Phys. Rev. Lett., 34, 1548-1551 (1975).

Sternberg, N. and V. Godyak. The Bohm Plasma-Sheath Model and the Bohm Criterion Revisited, IEEE Transaction on Plasma Science, 35, 1341-1349 (2007).

Vinogradov, G. K., Recent Trends in 300-MM Plasma Equipment, IEEE Transactions on Plasma Science, Eighth International Symposium on Plasma and Process-Induced Damage, 24-25, 168-173 (2003).

Yin, Y., J. Messier, and J. Hopwood, Miniaturization of Inductively Coupled Plasma Sources, IEEE Transactions on Plasma Science, 27, 1516-1524 (1999). 